\title{
GREENIE: a novel hybrid routing protocol for efficient video streaming over wireless mesh networks
}

\author{
Behrang Barekatain ${ }^{1 *}$, Mohd Aizaini Maarof ${ }^{1}$, Alfonso Ariza Quintana ${ }^{2}$ and Alicia Triviño Cabrera ${ }^{3}$
}

\begin{abstract}
In recent years, video streaming over wireless mesh networks (WMNs) has been of great interest among the users. In WMNs, although node mobility and scalability are the two most important well-known advantages by end-users, they can decrease the perceived video quality on receivers with increasing the probability of path failure, especially when the number of mobile mesh nodes and their mobility speeds increase. Therefore, the necessity of employing an efficient routing protocol to consider the effects of node mobility is inevitable. Moreover, the interference can be sharply increased, especially on the gateways, when there are many mobile mesh nodes in a WMN. Interference does not permit the system to support many STA and mobile mesh nodes. In order to cope with these challenges, this study introduces and evaluates a good hybrid routing protocol for data dissemination which efficiently and effectively routes packets in a wireless mesh network and intelligently employs proactive and reactive routing protocols based on the node mobility (GREENIE) for efficient video streaming over WMNs and extensively compares it with other routing protocols including hybrid wireless mesh protocol, proactive, reactive, and spanning trees using OMNET++ simulator. GREENIE intelligently distinguishes mobile from static nodes and selects the most stable path between a source and a destination which leads to higher perceived video quality on receivers. The results show the impact of GREENIE on the perceived video quality so that it considerably outperforms other routing protocols in terms of the total number of successfully received packets, the end-to-end delay, and the imposed routing overhead on the system. One of the main advantages of GREENIE is that it performs routing in the medium access control layer without applying any change in the functions of the internet protocol layer.
\end{abstract}

Keywords: Hybrid routing protocol, WMNs, Video streaming, Quality-of-service

\section{Introduction}

A wireless mesh network (WMN) is a hybrid network built on a mix of fixed and mobile nodes which are interconnected via wireless links [1,2]. WMN is a subset of ad hoc networks [3] and is envisioned as the economically viable networking paradigm to build up broadband and largescale wireless commodity networks. In contrast to traditional wireless local area network (LAN), which can only cover an area of up to hundreds of meters, WMNs can cover practically tens of kilometers [1,4]. This ability has removed the huge costs associated with the provision of expensive infrastructures to enable wide coverage, which

\footnotetext{
* Correspondence: Bbehrang3@live.utm.my

${ }^{1}$ Faculty of Computing, Universiti Teknologi Malaysia, Johor Bahru 81310, Malaysia

Full list of author information is available at the end of the article
}

is an important requirement in recent years. WMN also removes the constraint of a wired infrastructure. Thus, a wireless mesh network can be easily deployed in hard-towire areas, in difficult terrains, in monuments, or in those areas where environmental concerns prohibit wiring. The scenarios where a WMN can be deployed also include cities or commercial areas [5]. In these situations, some nodes in the WMNs are usually provided with a wired backbone to allow any device in the network to access the Internet. Considering the IEEE $802.11 \mathrm{~s}$ standard [4], a WMN has some fixed elements and some mobile nodes. The fixed elements or mesh routers (MR) create a backbone. MRs are mesh points (MP), mesh access points (MAP), and mesh portal points (MPPs) [6]. A MP is a device that communicates with its peer to make a 
forwarding path. It is only used for one hop communication to create a peer link.

On the other hand, a MAP is a mesh point that additionally supports non-mesh nodes or STA nodes. In this way, a node that does not implement any mesh routing protocol can gain access to the WMN. The STA nodes usually have a low mobility degree. The MAP can also allow an internet connection to those non-mesh nodes if the WMN is provided with MPPs. A MPP is a MR that acts as an exit and entry point for data which need to travel outside the mesh or vice versa. Referred as gateways, the MPPs can also connect to the wired Internet, to a static server, or to any other LAN infrastructures. Taking the performance into account, WMNs provide wider coverage area and better performance in comparison with WLAN, wireless metropolitan area network (WMAN), and ad-hoc networks [6]. These advantages are mainly due to the capability of a WMN to create optimal forwarding paths, which introduce enough redundancy in the system to make it less prone to failures. Basing on a multi-hop routing paradigm, the paths are composed of multiple nodes. The WMN autonomously discovers and repairs the communication paths.

Although WMNs increase data communication performance using the multi-hop technique [7], the overall performance of the network sharply decreases if the number of nodes, especially mobile mesh nodes, increases. Due to this limitation, WMNs are suitable for light-traffic applications such as email services. However, they are not appropriate for high-traffic and delay-sensitive applications such as video conferencing. This drawback is especially notable when different live streams traverse some common links. Moreover, local congestion due to the traffic dynamic nature and interflow interferences significantly degrades the perceived video quality on receivers $[8,9]$. In summary, the dynamic network conditions such as number of hops between the source and destination, link stress, contention on the limited available resources, network size, mobility rate, number of nodes, and routing polices have considerable effects on the video quality perceived by the WMN receivers. Therefore, it is necessary to design efficient routing protocols that simultaneously reduce the delays and the losses. Towards this goal, this paper proposes a new hybrid routing protocol which promotes the use of the stable paths in the WMN. The protocol is named GREENIE. GREENIE is a good hybrid routing protocol for data dissemination which efficiently and effectively routes packets in a wireless mesh network and intelligently employs proactive and reactive routing protocols based on the node mobility. In contrast to previous works, the protocol gives priority to all the stable routes independently of the fact that the gateway is part of the route or not. In this sense, the intra-mesh traffic will be improved.
In contrast to ad hoc on-demand distance vectorspanning tree (AODV-ST) [10], GREENIE assumes that a stable path is the concatenation of links formed among static routers. The stable paths, which are expected to endure longer, are discovered and maintained by a proactive routing scheme. Conversely, other links are only used and discovered on demand. The main features of the protocol are the following: (1) it combines two routing schemes in the MRs; (2) routing procedures are transferred to the link layer; (3) the reactive route discovery process is adapted to promote the use of static routers; and (4) it supports mobile STAs. The efficiency of the protocol has been tested analyzing the performance of video streaming in a WMN. The characteristics of the tested network have been varied so that a comprehensive set of networks has been used in the evaluation process. The evaluation has been carried out using the OMNeT++ simulation tool, which needs to be adapted to support the proposed protocol.

The rest of this paper is organized as follows: video streaming and routing protocols in wireless mesh networks are explained in Sections 2 and 3, respectively. Section 4 provides an overview over the most recent related works, whereas the problem statement is propounded in Section 5. GREENIE, the proposed hybrid routing protocol, and the results with $95 \%$ confidence interval are explained in great details in Sections 6 and 7 , respectively. Finally, remaining issues and future works are discussed in Section 8, and the paper is concluded in Section 9.

\section{Video streaming}

Video streaming over computer networks is considered to become the most interesting application in the near future [11]. This kind of application needs large bandwidth, efficient routing protocols, and content delivery methods to provide smooth video playback to the receivers. Video streaming systems are classified into two categories including live and video on demand (VoD) [12]. Some technical differences exist between live and VoD streaming. In live video streaming, synchronized streams are played back in all nodes, and all users watch the same video frames simultaneously. However, in VoD streaming, users watch different video frames of the same video stream at a given instant of time. In other words, the playbacks of the same video streams on different clients are not synchronized for a VoD streaming.

In addition to enough large bandwidth, efficient delivery protocols, buffering techniques, and a suitable video compression standard also help to increase the video quality. The H.264/advance video coding (AVC) [13], which is an open-licensed standard, is one of the most recently used video compression standards in video streaming applications. This standard is based on different existing profiles 
and levels. In particular, several orders of frames can be encoded into a group of picture (GOP). Each GOP consists of frames I, B, and P as shown in Figure 1. The solid lines in this figure show decoding dependencies among video frames. For example, the successful decoding of all frames is dependent on receiving frame I. Video streams can be encoded using constant bit rate (CBR) or variable bit rate (VBR) [14] techniques. Contrary to CBR technique which encodes the video frames with a predefined data rate, VBR encoding adjusts the data rate between a minimum and a maximum value according to the required compression rate. The CBR technique is suitable for video streams with the same motion degree during the whole video playback, whereas VBR method is commonly used for video delivery over hyper text transfer protocol (HTTP). Here, this study refers interested researchers in video encoding and compression standards to [13] for more useful information.

\section{Routing protocols in wireless mesh networks}

The challenge of a routing protocol is selecting the optimum or minimal path to communicate the source (e.g., the video server) and the destination (e.g., the mesh client). Taking advantage from the static backbone, the focus of routing protocols for WMNs should be on achieving higher throughput rather than saving energy or improving resiliency for mobility [15]. There are three general categories of routing protocols namely: reactive, proactive, and hybrid [16-18]. Reactive protocols only search for a path between two nodes when there are data to send. This method has the advantage of not wasting network bandwidth with control messages when data transmission is not required. Reactive protocols are ideally suited to an ad hoc network with mobile nodes where data path may change continuously whereas proactive protocols actively establish and maintain data paths for nodes whether data are needed to be transferred or not. The advantage of this kind of protocol is a low latency in sending data through the network since an optimized data path is already known before the transmission. Nevertheless, this comes at a price in terms of higher cost of network overhead associated to both network control messages and computational processing.

Considering the scalability, reactive routing protocols are designed to reduce the memory resources necessary for storing the routing information. The reduction is achieved because only the active routes are determined and maintained in the nodes. In addition, the route discovery in a reactive protocol usually occurs by flooding a route request packet through the network. Although the route discovery process happens more often in the reactive protocols, this process requires light control overhead traffic compared to the proactive routing algorithm. Hence, the reactive routing is considered to be more scalable than the proactive routing [19]. The following are examples of proactive routing protocols: destination-sequenced distance vector (DSDV) [20], better approach to mobile ad-hoc networking (BATMAN) [21], and optimized link state routing (OLSR) [22]. Examples of reactive routing protocols include dynamic source routing (DSR) [23], ad-hoc on-demand distance-vector (AODV) [24], and associatively-based routing (ABR) [25].

As a better solution, hybrid routing protocols exhibit both reactive and proactive properties [26]. Such protocols generally attempt to use reactive and proactive routing depending on different circumstances. For instance, the selection of the kind of routing protocol to use may be based on the distance of the destination or its mobility degree. In this way, the hybrid routing protocols aim at exploiting both routing strengths and hence can result in higher levels of scalability [27]. Hybrid wireless mesh protocol (HWMP) [28] is introduced as the default and the mandatory routing protocol in WMNs by the IEEE $802.11 \mathrm{~s}$ standard. It is a hybrid scheme based on AODV and the tree-based routing protocols. In AODV, routing tables are maintained by each node. They store the destination and the next hop internet protocol (IP) addresses as well as the destination sequence numbers. Each entry in the routing table has a destination address, a next hop, a precursor node list, a lifetime, and the distance to destination (measured as the number of hops). Route request (RREQ) packets are generated in order to initiate a route discovery process. The packet contains the source node's IP address as well as the destination's IP address. Once a RREQ is received by a node, it determines if it is the indicated destination. In this sense, it will reply with a route reply (RREP) to the source and the path will be established.

It is necessary to mention that flat routing protocols are commonly used in ad hoc networks. In contrary to cluster-based schemes, in these kinds of protocols, all the nodes are assigned the same routing tasks. These

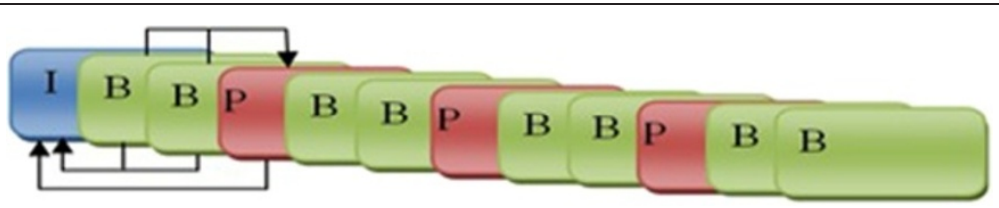

Figure 1 A standard GoP based on G12B2 structure. 
protocols can either belong to proactive or reactive routing protocols depending on their design philosophy as illustrated in Figure 2. Table 1 summarizes the characteristics of flat routing protocols based on the performed study in [29]. $\mathrm{N}$ and $\mathrm{e}$ in this table indicate the number of nodes and communication pairs in the network, respectively.

Altogether, many previous studies such as $[2,6,7,17,18,30,31]$ performed extensive surveys on different routing protocols for WMNs. Moreover, previous studies such as [32] performed a complete survey of quality-of-service (QoS) routing protocols. Therefore, this study refers interested researchers to such studies for more information about these routing protocols. Actually, the main focus of this study is to introduce a new hybrid routing protocol in WMNs and compare it with HWMP and reactive, proactive, and spanning tree routing protocols for presenting its comprehensive evaluation when video applications are active.

\section{Related works}

In order to have a precise overview of the previous studies, this section is divided into three subsections. Recent studies on hybrid routing protocols and video streaming over WMNs are discussed in Sections 4.1 and 4.2, respectively. Other related works are presented in Section 4.3.

\subsection{Recent proposed hybrid routing protocols}

Although many previous studies such as [33-36] evaluated the performance of existing routing protocols in ad hoc and wireless mesh networks, few of them considered hybrid methods, especially for video streaming applications. A neural network-based hybrid scheme is introduced by Nenad [37] based on both proactive and reactive routing protocols and mobile agent technology which is controlled by a Hopfield neural network. The Hopfield neural network is based on some interconnected neurons in which they independently and asynchronously update their activation values. The imposed complexity due to using such method is not cleared in this study. For example, the effects of using this approach on a delay sensitive video stream need to be studied. Actually, Hopfield neural networks need appropriate hardware which is not accessible on current gadgets such as Smartphones. Although Hopfield networks are really simple in implementation, they can produce fake data. Moreover, this approach requires more complex nodes and processing elements [38].

On the other hand, AODV and the spanning tree routing protocols are integrated together in AODV-ST [39] as a hybrid routing protocol. In the proposed hybrid protocol, the spanning tree protocol is used for finding routes to all existing gateways, whereas all nodes use AODV to establish a path among themselves. In contrary to the performed study by Ramachandran [39], the proposed hybrid routing protocol by Le [40] considers the mobility behaviors of nodes where selecting the routing method. Actually, it uses proactive mode to find the best possible path between a gateway and a fixed router, while the reactive routing protocol is used on mobile nodes. The main advantage of this hybrid routing protocol is that mobile nodes ignore RREQ messages which are periodically sent by gateways in order to construct the required spanning tree. In this regard, the established path between a node and a gateway consists of static nodes which lead to efficient data dissemination among them. However, the proactive routing exclusively builds proactive routes to the gateways. Our proposed routing protocol extends this approach by allowing the proactive discovery of any route formed by static routers, irrespective of this fact that the gateway is a node on the path or not.

Another hybrid routing protocol is proposed by Amir for multi-homed WMNs. It employs continual connectivity among mesh nodes using a fast handoff mechanism [41]. The proposed hybrid method integrates wired and wireless connectivity for TCP and UDP data transmission through gateways. The introduced hybrid routing protocol by Peppas considers heterogeneous mobility for mobile nodes [42]. Therefore, the protocol pays attention to the

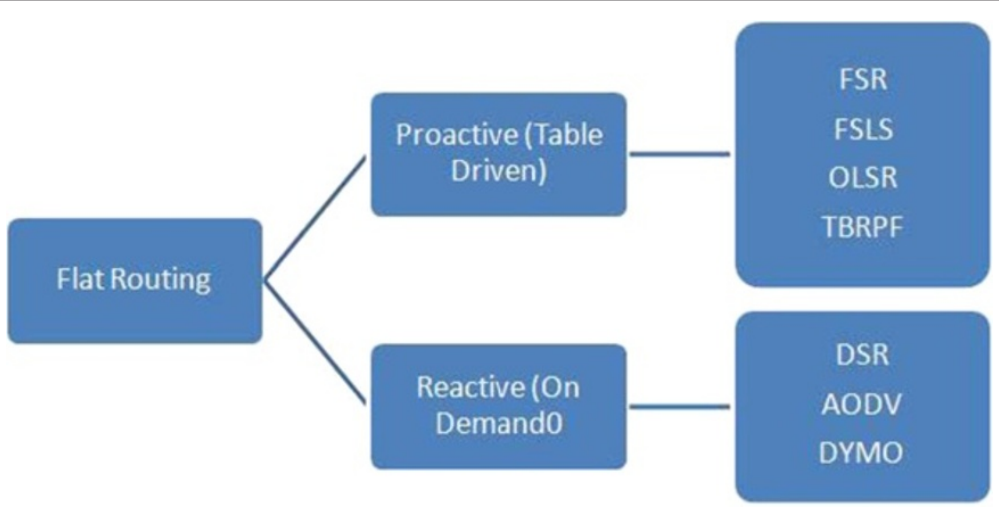

Figure 2 Flat routing characterization. 
Table 1 Characteristics of flat routing protocols

\begin{tabular}{|c|c|c|c|c|c|}
\hline Characteristic & FSR & OLSR & TBRPF & AODV & DSR \\
\hline Routing philosophy & Proactive & Proactive & Proactive & On-demand & On-demand \\
\hline Routing metrics & Shortest path & Shortest path & Shortest path & Shortest path & Shortest path \\
\hline Frequency of updates & Periodically & Periodically & Periodically as needed (link changes) & As needed (data traffic) & As needed (data traffic) \\
\hline Use sequence numbers & Yes & Yes & Yes (Hello) & Yes & Yes \\
\hline Loop free & Yes & Yes (pure LS) & Yes & Yes (full flooding) & Yes (full flooding) \\
\hline Worst case exists & No & Yes & No & Yes & Yes \\
\hline Multiple paths & Yes & No & No & No & Yes \\
\hline Storage complexity & $\mathrm{O}(\mathrm{N})$ & $\mathrm{O}(\mathrm{N})$ & $\mathrm{O}(\mathrm{N})$ & $\mathrm{O}(\mathrm{e})$ & $\mathrm{O}(\mathrm{e})$ \\
\hline Comm. complexity & $\mathrm{O}(\mathrm{N})$ & $\mathrm{O}(\mathrm{N})$ & $\mathrm{O}(\mathrm{N})$ & $O(2 N)$ & $\mathrm{O}(2 \mathrm{~N})$ \\
\hline
\end{tabular}

mobility behaviors of nodes so that terrestrial and aerial nodes employ proactive and reactive modes, respectively. However, although this hybrid protocol is similar to GREENIE when classifying the nodes based on their mobility behaviors, it can be used just for unmanned aerial vehicles (UAV) systems. Thus, it is not applicable for WMNs. In fact, the aerial nodes can access to the WMN through some specific mesh routers. Furthermore, there are some other issues in WMNs such as the node density and high interference which are not considered in it. In hybrid on-demand distance vector routing (HOVER) [43], a non-optimal path between a source and a destination can be quickly established, then the system tries to find a better path. The results of the HOVER show that it improves the latency and packet delivery delay in hybrid WMNs using a modified version of AODV which estimates link quality using HELLO messages. A new routing metric is proposed for the reactive discovery. The new metric differentiates among node types (static or mobile). The protocol also defines a channel selection with minimum interference in multi-radio WMNs. However, HOVER does not take into consideration the mobility behavior of nodes and it uses the same routing scheme for both static and mobile nodes. Thus, the stable routes are discovered and maintained reactively. This behavior is not recommended in other works such as [7].

Another hybrid routing protocol is introduced by Zhang for VoIP traffic transmissions [44]. It includes both network and link layer routing and is based on intra- and inter-domain mobility management methods. New routing information can be found using address resolution protocol (ARP) messages during intra-domain handoff to prevent location update and re-routing, while the introduced latency is decreased by removing the redundant tunnel for inter-domain handoff. In these proposals, the imposed complexity by intra-domain and inter-domain handoff has not been studied at all. This cost is expected to be significant. In addition, the protocol needs to send many different messages such as the association control, GARP, and the link control messages. Therefore, the handoff overhead sharply increases when the mobility rate and speed increase. The previous proposals are not specifically designed to support STA nodes. Moreover, they usually use infrastructure WMN, and there is no multi-hop communication path in the mobile components. In this solution, although the packets forwarded among the mesh routers can use link layer routing to decrease the encapsulation and decapsulation delay associated to IP datagram, the packets exchange among access routers and mesh clients rely upon the network layer routing. Thus, they inherit the disadvantages of performing routing in the IP layer. In contrast, GREENIE does not use the IP layer for routing at all neither on ARs nor on the wireless clients.

\subsection{Video streaming over WMNs}

Considering the assumed traffic in our study, in the following, some recent works on video streaming over WMNs are described. An evaluation of video streaming over WMNs is performed and the results can help other researchers to understand the efficiency of multi-hop WMN in video dissemination [45]. In recent years, a lot of improvements have been experienced in video streaming over multi-hop wireless mesh networks, which results in many commercial products and academic research findings. Some of these works such as [46] strived to provide better video quality on receivers by modifying the medium access control (MAC) layer, whereas others introduced efficient solutions in the application layer. Altogether, these research findings can be categorized into three categories.

The first category focuses on cache-based approaches. A simple method for increasing perceived video quality on wireless nodes is caching video frames in mesh nodes and MRs. Therefore, each node can request required video frames by sending a search message to its neighbors. This strategy decreases the load on the gateways. Basically, this approach is suitable when there are many nodes in the network and each of them has a significant capacity to save received video frames. In Ditto [47], the broadcast nature of WMNs is exploited in order to increase the total throughput of the system so that the mesh nodes overhear video chunks. The operation of Ditto is 
similar to hierarchical web caching where each node caches and sends data to the next hop. However, it increases the throughput of the wireless network up to seven times more than simple on-path caching. Ditto considers a 3-GB cache size on each node. An important result of the proposed system is that the size of the video chunks has enormous effects on the performance of the network so that transferring video chunks with smaller size provides better throughput.

In contrary to Ditto, APPCCM [48] lets MRs and mobile mesh nodes cache video chunks in order to accelerate data access for mesh nodes and decrease the load on the gateways. In fact, the proposed model makes a precise decision about where to cache a video chunk basing on its two caching mechanisms: client-cache mechanism (CCM) and data-proxy mechanism (DPM). UPAC [49] is a Unified P2P and cache-based framework for VoD dissemination over multi-hop WMNs. Some selected MRs cache the received video frames. Then, each peer establishes a P2P relationship with a MR and fetches cached frames from it using a client-server-based communication. Cache-based approaches are not cost effective due to highly required capacity in each node and maintenance.

Bandwidth-traffic-based approaches are used in the second category. One of the most efficient approaches to decrease load on the mesh routers and on the gateways is to reduce the wireless traffic. Although cachebased approaches reduce the traffic by caching just the received video frames, they cannot save limited valuable resources in WMNs efficiently. A dynamic stream merge (DSM) method is introduced by Nichols [50] in order to address this problem. The main idea in DSM is to merge at least two similar streams in an intermediate node. A bandwidth-traffic-based method needs intelligent algorithms in order to efficiently assign bandwidth to data streams. For example, these solutions can be topology dependent when a node needs to receive at least two similar streams in order to merge them as one stream. Exclusive OR (XoR) network coding [51] is one of the most popular bandwidth-traffic-based approaches. In this method, each intermediate node tries to apply XoR logical operation on the received video packets in order to send one encoded packet instead of many original video packets. Although this method can be effective, it is both topology dependent and needs an intelligent algorithm to encode those video packets which can be decoded in the maximum number of receivers.

The third category is based on the path selection approaches. Here, each node selects the best path to the destination based on some parameters such as delay, jitter, packet loss rate, and throughput of the links. A grey relational analysis (GRA)-based [52] approach is proposed by Razzaq [53] for selecting the best path for the most important layers of a SVC stream. Minimum interference route selection (MIROSE) [21] is an enhanced network route selection method which finds the best path with the least interference for video dissemination. A video agent, which is embedded in the access points between the mesh network and the Internet, receives video frame requests from all mesh nodes and chooses a proper path with minimum path contention to send the video flow to them. Moreover, this agent buffers the video stream and adjusts the compression rate regarding the condition of the path between each mesh node and the gateway using network status-dependent video compression rate (NSDVCR) algorithm. However, MIROSE imposes a source-routing strategy, which is not possible according to the IEEE $802.11 \mathrm{~s}$ standard. Furthermore, the mobility of the source will report a tremendous cost when recomputing the communication paths.

The most significant drawback concerning the previous proposals is that they are specifically designed for video traffic. Other kinds of traffics with different features may get a worse performance because of this adaptation. In particular, the routes used to send the video frames are those that are expected to suffer from a lower number of losses. In this sense, the links established among fixed nodes are preferred to the links with mobile routers. The fixed links can offer a higher throughput as they are not so affected by the disturbance of the mobile elements. In this way, the video quality is improved. Our proposed algorithm follows the last approach but in more realistic routing conditions that makes it appropriate for different kinds of traffics (web surfing, video streaming, email access).

\subsection{Other related works}

Some recent studies such as $[41,54]$ have employed the existing gateways in WMNs to transfer some of the video frames generated by the wireless nodes to the wired gateway links. The main idea behind these studies is that the data transmission through the gateways and the existing internet connection provides higher video quality with lower end-to-end delay due to employing high capacity bandwidth over the Internet in comparison with wireless network. Although the obtained results are interesting, data transmission through public networks (e.g., the Internet) introduces some new issues such as data communication security. Moreover, it is very important to use intelligent algorithms when selecting a gateway, because the probability of congestion and single point-offailure can be increased using gateway connections. One of the most recent research areas in the high quality multimedia dissemination over WMNs is the efficient utilization of the existing network resources in wireless mesh networks. In this sense, it is possible to have many concurrent multimedia flows, especially when the channels become saturated. Two policies including flow scheduling 
and channel aggregation are studied in both multi- and single-hop wireless networks [55]. Then, the author introduced the efficient multi-flow multicast transmission (EMMT) algorithm to apply the mentioned policies. The results show that more multimedia flows can pass through the channels. Finally, an on-demand channel reservation scheme is proposed by Mogaibe for common traffics in order to improve the performance of multi-channel multiradio WMNs [56]. This scheme uses the mesh routers radio interfaces for gateway and local traffic effectively so that intra- and inter-flow interference decrease. Obviously, if the number of nodes which share a channel decreases, the throughput of that channel will be maximized. This is the same for the gateways. Equation 1 clearly shows this assertion. Here, node $v$ has $n(v)$ neighbors and $n(x)$ number of them have some traffics through a gateway, while $n(y)$ of them have just local traffics. These nodes use channel $d$ which is a member of $C$ non-overlapping channels. In this equation, $f_{\mathrm{t}}$ is the throughput function of channel $d$. As can be seen, the maximum number of nodes which have gateway traffics has a direct relationship with the minimum number of those which have local traffics.

$$
\begin{aligned}
& d_{\max }(n(x))=\arg \underset{d \in C}{\operatorname{Max}_{d}} T_{d}(n(x))=\underset{d \in C}{\operatorname{Min}} n(y), \\
& \text { where } T_{\mathrm{d}}(n(x))=\frac{n(x)}{n(x)+n(y)} f_{\mathrm{t}}(n(x)+n(y)) \\
& \text { and } n(x), n(y) \in n(v) d \in C
\end{aligned}
$$

\section{Problem statement}

Recent studies show that the performance of routing has been improved by either improving the efficiencies of the current routing protocols or introducing new hybrid methods. However, there are some unresolved issues in efficient data streaming over WMNs such as the following ones:

1. The performance of a proactive routing protocol considerably decreases in very dynamic networks. In other words, proactive routing protocols select the best possible path based on the pre-calculated routes and some routing metrics. When a source node sends a packet to a destination and a link on the considered path fails, the packet will be undoubtedly lost. Hence, this type of routing protocol is not suitable for dynamic networks where links fail or nodes move continually. High path failure rate considerably degrades the video quality on nodes.

2. Contrary to the proactive routing protocol, reactive routing protocols are less suitable for non-dynamic networks. In fact, they are more suitable for dynamic scenarios where there are mobile nodes in the network and the probability of link failure is not negligible [57].

3. Certainly, it is not expected that wireless mesh networks present the same behavior along their operation times. Mobility, as mentioned before, is the most attractive feature of wireless networks for end users. However, it causes many problems such as high link failures due to missing neighbors. Due to the mobility and the traffic of the nodes, the conditions of the network vary dynamically. Moreover, time-varying channels considerably heighten this problem. The mentioned problems have been tackled in some previous studies to introduce new protocols or enhance the existing hybrid routing protocols to exploit the benefits of both proactive and reactive schemes simultaneously. However, they inherit the existing challenges in proactive and reactive protocols. Actually, the problem is that they use proactive or reactive protocols in both mobile and fixed (static) nodes simultaneously $[1,40]$ without paying enough attention to their mobility behaviors. Hence, the side effects of proactively broadcasting RREQ in mobile nodes in a high dynamic network cause new challenges to the system such as high traffic, waste of available bandwidth, and more interferences. On the other hand, in fixed nodes, employing reactive protocols leads to perform many routing operations for finding the next hop which means a huge amount of resources will be wasted. As a result, current hybrid routing protocols cannot completely and effectively exploit the benefits of proactive and reactive protocols, because they cannot precisely distinguish between fixed and mobile nodes. In order to clarify this problem, suppose that there are four nodes in the network: nodes $\mathrm{A}$ and $\mathrm{B}$ are fixed whereas nodes $\mathrm{C}$ and $\mathrm{D}$ are mobile. Hence, there is no signal difference among links $\mathrm{A}-\mathrm{B}, \mathrm{C}-\mathrm{D}$, and $\mathrm{A}-\mathrm{C}$ using the current hybrid routing protocols.

4. Any change in the IP layer leads to a high cost into the system. Thus, those routing hybrid protocols which try to change the functions of the IP layer to introduce better routing protocols cause other challenges such as high cost to the system. Consequently, it is better to transfer the routing operation to the MAC layer.

5. Mobility is one of the most important factors which needs to be considered by all routing protocols in WMNs. In fact, the time-varying links of the mobile devices have considerable effects on the network performance. Therefore, considering the same routing protocol for mobile and fixed nodes causes a low performance in the 
network. Moreover, those routing protocols which just try to find a path to the destination without paying attention to the mobility behaviors of the intermediate nodes on the path cannot guarantee efficient end-to-end data transmission, because the mobility behavior of the intermediate nodes has also noticeable effects on the probability of path failure.

6. The use of a routing protocol specifically designed to support only a kind of application restricts the operability of the network. Particularly, WMNs are expected to be used for heterogeneous applications such as video streaming, web navigation, or email access.

Based on the mentioned challenges and issues, this study aims to introduce an efficient hybrid routing protocol which completely considers these challenges and provides noticeable performance in both low and high dynamic scenarios. Section 6 will explain GREENIE, the proposed hybrid routing protocol, in more detail.

\section{GREENIE: the proposed hybrid routing protocol}

As mentioned in the previous sections, there are many different routing protocols for discovering and maintaining paths among the components of the WMN. The convenience of using one of these types of protocols mainly depends on the communication path lifetimes. The path lifetime or route duration is impacted by the relative mobility of the intermediate nodes from the source to the destination [58]. Taking into account this behavior, GREENIE carefully considers the mobility of the nodes in order to associate the different routing tasks to the WMN nodes. In particular, most stable routes, that is, the routes that are expected to endure longer are periodically updated. In contrast, the unstable routes are only discovered on demand. Conversely to other mentioned proposals, GREENIE does not exclusively promote the routes to the gateway but all the routes composed of static routers. Among the routes to which GREENIE gives priority, the paths to the gateway are also included. However, these routes to the gateway are restricted to the links established between two fixed elements. If we compare GREENIE with AODV-ST, GREENIE shortens the routes which are proactively discovered because mobile links are not considered. In the following, the main features of GREENIE are discussed.

\subsection{The assignation of the routing tasks depending on the node mobility}

The mesh routers, which are fixed, employ two types of routing protocols including proactive and reactive simultaneously. They exchange their routing tables among themselves using the proactive routing protocol. Thus, the backbone is restricted to use just the proactive mode. The main idea is that the conditions of links among MRs do not change drastically so that it is better to use a proactive routing protocol in a stable topology as it occurs in the backbone. On the other hand, the conditions of a link established between a mobile node and a MR or between two mobile nodes can change unpredictably and significantly. In fact, routes from a mobile node to the MRs or to the gateways or between any two mobile nodes in the hybrid WMN may include non-stable links. In this regard, a reactive scheme is appropriate for this kind of communication paths. Thus, the static (fixed) mesh nodes, like MRs, run both schemes, while mobile mesh nodes employ a reactive mode. The use of a reactive protocol in the MR even when they are already designed with the proactive one guarantees the compatibility with the mobile nodes. The use of a reactive routing protocol in the mobile nodes provides an efficient self-healing method because the link-layer feedback has been activated. In this sense, the nodes can efficiently identify when an active link to a node is broken.

\subsection{Implementation in the MAC layer}

In GREENIE, both proactive and reactive routing protocols are implemented in the MAC layer of each MR and node. The transfer of the routing procedures to the link layer is necessary since the mesh routers need to run both schemes simultaneously. In contrary to the IP layer, it is possible to use two different independent forwarding tables in the MAC layer. There are some benefits of transferring the routing task from the IP layer to the MAC layer. First, this allows an MR to be more efficient for routing the received packets in the WMN. Since the link layer forwarding tables do not contain any information about any node outside the local network, the memory resources are smaller. Consequently, the time resulting from accessing the table is also decreased. Moreover, there is less overhead in the routing frames. Second, less IP addresses are required. This leads to support a higher number of mesh nodes in the network. Finally, it is possible to use the existing information in the MAC header in order to update the route information to the source directly and efficiently. The MAC layer does not exclusively inform about the destination but also includes information about the previous node. Thus, the receiving node can update the routing information concerning the previous node. The promiscuous mode is also a possibility to refresh the routing information without any additional cost. Thus, GREENIE is developed and implemented in the MAC layer. This removes the need for any change in the IP layer as it occurs in the performed study by $\mathrm{Fu}[59]$. 


\subsection{Integration of the proactive and reactive information in the mesh routers}

The previous hybrid proposals clearly use a classification of the destination nodes in the source. Thus, when the source needs to know a route to a destination, it already identifies in which routing structure it should look for it. This condition does not hold in GREENIE, because the proactive and the reactive forwarding tables may contain the information associated with the same destination node. GREENIE promotes the use of the most stable links so that the routing information kept in the proactive routing table is preferred.

GREENIE uses the proactive and reactive modes derived from the OLSR and the AODV/DYMO [60] routing protocols, respectively. When a static node receives a frame, it first searches in its proactive routing table for a valid path. If it is found, the frame is sent according to the routing information. Otherwise, the proactive scheme transfers the responsibility of establishing a valid routing path to the reactive protocol. Then, the reactive routing protocol searches for a valid entry in its forwarding tables. If it is found, the reactive routing protocol can transmit the data frame. Alternatively, the reactive protocol triggers the corresponding procedures to discover a path to the destination. The destination or an intermediate node knowing the route to the destination may respond to the message with a RREP. Upon receipt, the source updates its routing table, and the frames are sent accordingly. In the data transmission, the bidirectional path is also set.

The RREQ messages generated by the reactive routing protocol as well as all the broadcast messages are generated with a sequence number. The sequence number helps a receiving node to detect the message whether it is new or it is a copy of a previous message. In this way, the receiving nodes maintain a table to associate the sequence number and the sources which are employing them. Thus, a receiving node only pays attention to the messages with a sequence number which is higher than the one stored for the source. In order to implement this behavior properly when managing multiple routing protocols, in GREENIE, a source just uses one sequence number which is incremented either by the reactive or the proactive routing protocol when a broadcast message needs to be generated.

Figure 3 clearly shows the route discovery process in more detail. In fact, according to the existing feedbacks between these protocols, if a mobile node wants to communicate with a fixed node, it will begin a search using the reactive protocol, and at the moment that the request route arrives to a fixed node, it knows a valid route thanks to the proactive protocol. Therefore, the fixed node answers immediately with a route replay packet. This mechanism lets selected paths include more fixed than mobile nodes which results in considerable performance in data delivery. Finally, a pure reactive search will only happen if the source and the destination are both mobile mesh nodes. These are the two other advantages of the hybrid routing protocol used in this study. In fact, an important advantage of using GREENIE is that it provides an efficient hybrid search method so that each node first searches a stable route using the proactive method and then non-stable routes using the reactive method. In other words, it prefers a path with more fixed nodes and static route to a path consists of more number of mobile mesh nodes. If there is no such a stable route, the node tries to find a new path to the desired destination using reactive method. This not only decreases the search time for the best path, but also provides higher averaged video quality among nodes thanks to the use of more stable links from the source to the destinations.

\subsection{Route discovery process modified to promote the use of MRs}

All the nodes in the WMN implement a reactive routing protocol, but the MRs also execute the proactive routing scheme. The MRs are able to respond to the RREQ messages even with the information derived from their proactive routing protocol. In fact, the routing data kept in the proactive tables should be preferred as they are associated to stable routes. In order to promote the use of the stable links, those established between two static routers, GREENIE sets different response intervals in the nodes. In particular, the MRs answer the RREQ messages with a random delay in the 0- to 5-ms interval. In contrast, the mobile nodes postpone their answers with a random delay following a uniform distribution function in the 5- to $10-\mathrm{ms}$ interval. It is necessary to include random delays in order to prevent the broadcast storming $[61,62]$.

\subsection{STA location management in the link layer}

As stated by Mase, the routing protocol in the WMN needs to know to which MR an active STA is attached [63]. Similarly, we have developed an agent-based scheme but at the MAC layer. This modification is necessary since the routing protocols run in this layer. By means of an internal data structure known as the localization table, the static, and the mobile routers know to which MR they should forward the packets whose destination is a STA. When the STA moves to another MR, the new MR alerts the other mesh routers about this new attachment so that they can update their localization table. The MR to which the STA is attached is also responsible for answering to the RREQ on behalf of the STA.

The use of the localization tables reports several advantages: firstly, the forwarding tables are completely independent of the number of STAs that the WMN is supporting. 


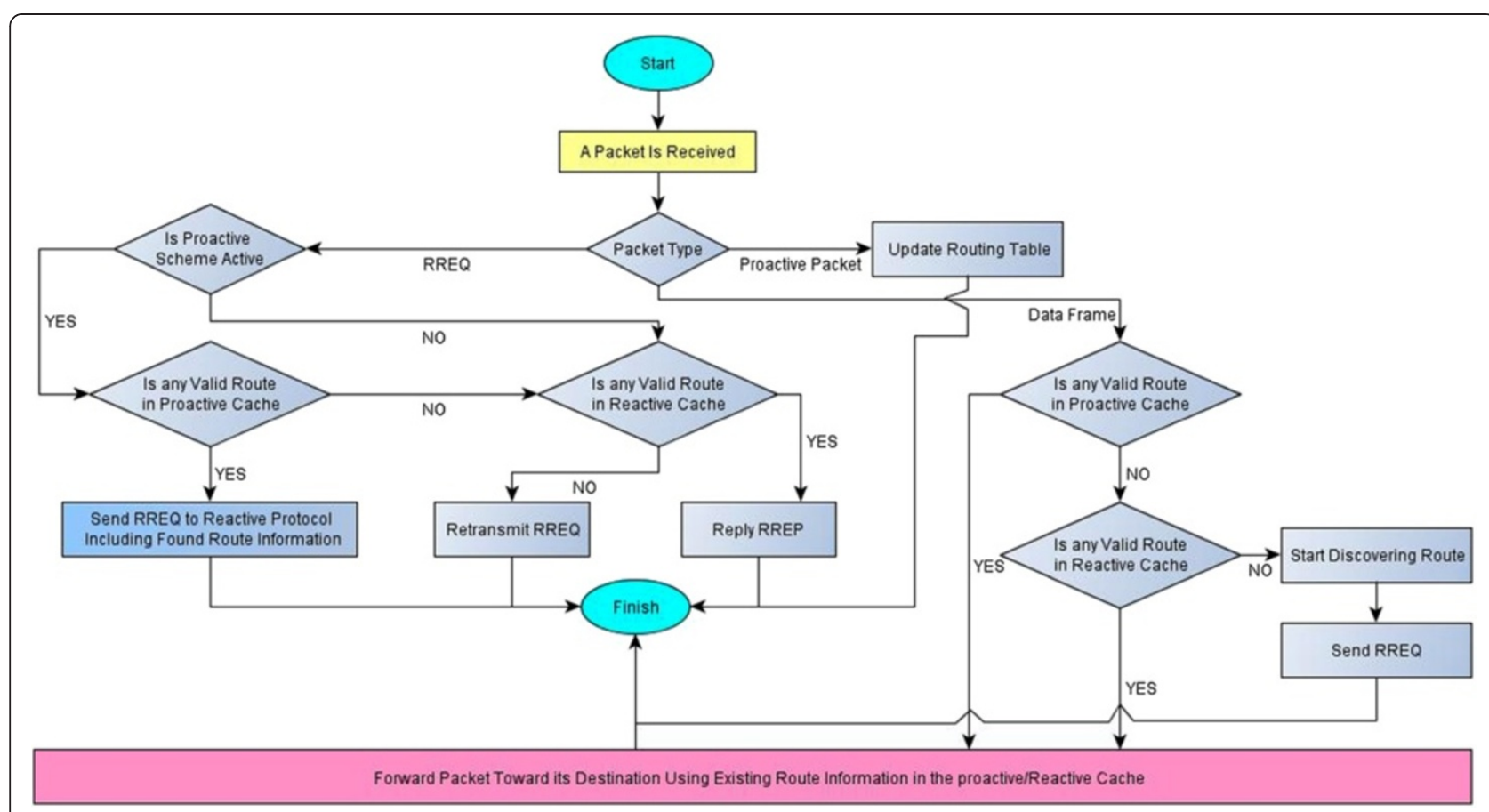

Figure 3 The operation of the GREENIE in a node.

The changes of the localization of the STAs are immediately announced. However, when the corresponding changes have not taken place, the MR to which the STA was previously attached is able to retransmit the frames to the new localization. This new localization is usually a neighbor so that the retransmissions do not incur in a high cost.

All in all, although previous studies introduced different hybrid routing protocols, the main advantage of the proposed hybrid routing protocol by this study (GREENIE) is that it logically divides existing nodes in the network based on their functionality. In other words, it generates two routing tables in static routers and fixed nodes. One of these routing tables is for proactive scheme supporting by OLSR, while AODV/DYMO, a reactive scheme, is used for generating the second one. In contrast to all previous proposals, GREENIE can intelligently distinguish between the existing link between two devices including mobilemesh-node $\leftrightarrow$ static-node, static-node $\leftrightarrow$ MR, and mobile-mesh-node $\leftrightarrow$ MR. Hence, it will select the most stable path including as many as possible static nodes. This does not only decrease the probability of path failure, but also results in better perceived video quality on the receivers by delivering more video packets to them using stable routes. Actually, these considerably degrade the effects of time-varying channels on the perceived video quality on receivers. In other words, it reduces the probability of applying any changes in the routing tables provoked by the time-varying channels due to the motility of the nodes.

\section{Simulation results and discussion}

In this section, the efficiency of GREENIE is evaluated and compared with the performances of four important routing protocols including HWMP, OLSR as a proactive routing protocol, and DYMO as reactive, and the spanning tree routing protocols. We disabled the path accumulation feature in DYMO. Therefore, it was treated like AODV. Moreover, the proactive spanning tree mechanism has been implemented by modifying DYMO. In this case, the root sends a RREQ packet. Then, all the intermediate nodes broadcast it and answer with a RREP at the same time. By comparing this protocol with OLSR and DYMO, we can confirm the benefits of combining both strategies in our proposed protocol. We have also evaluated our proposal against two of the most significant hybrid protocols, HWMP and the spanning tree.

In order to evaluate these protocols, a hybrid WMN is designed and implemented using INETMANET framework in OMNET++ [64] as shown in Figure 4. The INETMANET is based on the INET framework. However, in this work, it has been adapted to wireless networking. Moreover, different numbers of STA and 30 mobile mesh nodes are considered in this simulation in a $1,000 \times$ $630 \mathrm{~m}^{2}$ area. Mesh nodes move in the network continually with a speed of 5 to $20 \mathrm{mps}$ (meters per second), while STA nodes move with a speed of 1 to $2 \mathrm{mps}$, and they can stop in a position for a random time between 100 to $500 \mathrm{~s}$ before starting to move again. All STA nodes are connected to MRs, because they work in infrastructure mode and they are not able to route or forward the received traffic. 


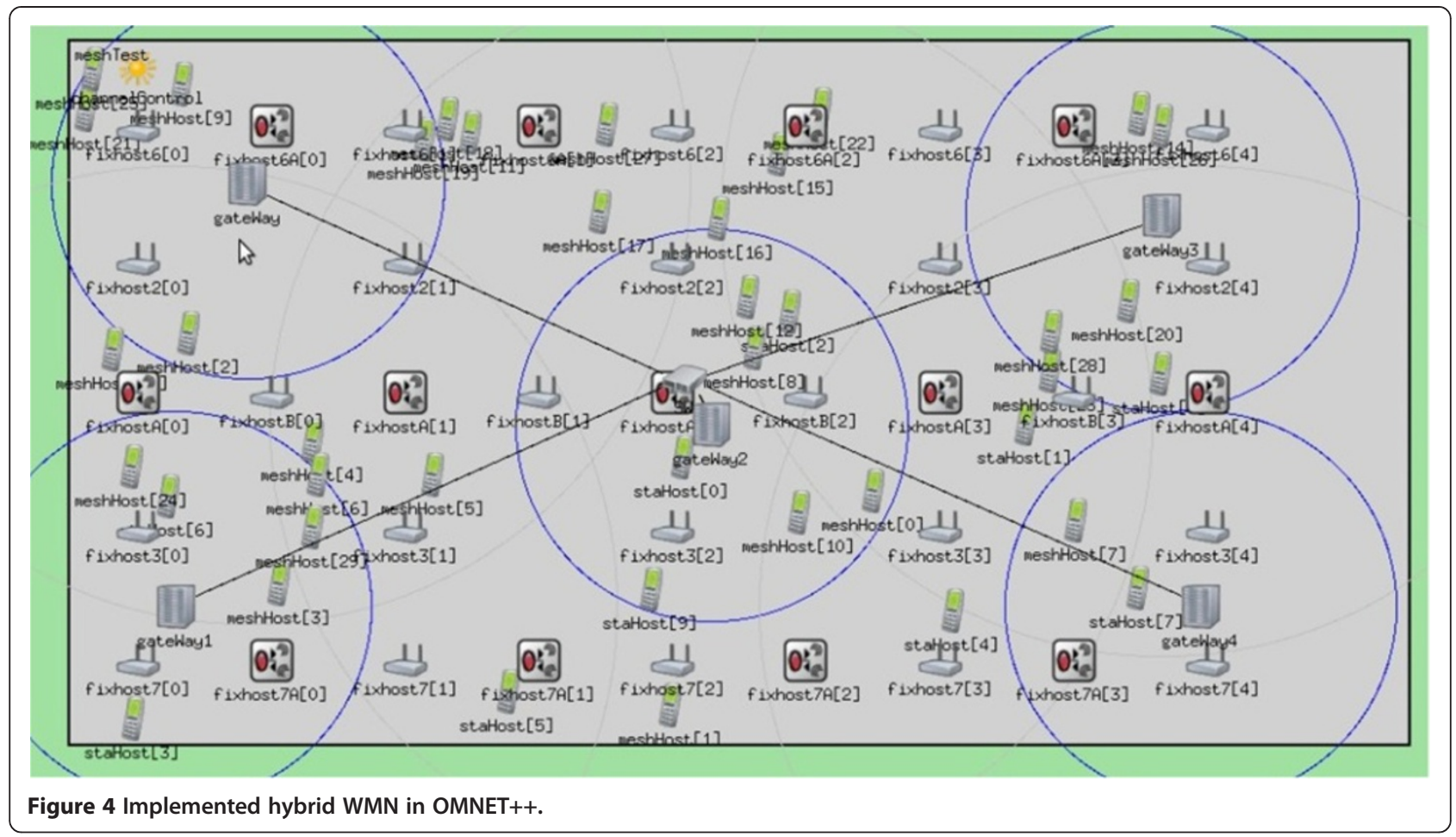

All required information about their positions in the network can be handled by the designed locator in this study. In addition to the employed STA and mobile mesh nodes, this study considered some static (fixed) nodes in the implemented hybrid WMN. The number of mesh nodes varies in the different experiments. These static nodes include mesh capabilities, but they cannot act as an access point. The main purpose of using them is to extend the network using their routing capabilities. The initial positions and the mobility models of the nodes are based on the uniform distribution and the random way point, respectively.

Concerning the traffic, a video server divides the video stream into some 512-byte packets and disseminates them among existing applicant nodes in the network. The video stream is encoded using the CBR technique. Table 2 summarizes all considered conditions and parameters in this simulation. This study selects video traffic due to its popularity; however, the application of GRENIEE is not restricted to video traffic.

The efficiencies of the considered routing protocols are evaluated in terms of three performance metrics including total number of successfully received video packets, endto-end delay, and routing overhead. The first metric indicates the averaged number of received video packets in the applicant nodes who have requested the video stream from the server. These packets are received on receivers with no error. The second metric, end-to-end delay, states the transmission time between sending and receiving a video packets in the video server and an applicant node, respectively. Moreover, we measured the amount of jitter for all considered routing protocols. The results show that both GREENIE and proactive introduced the lowest amount of jitters, while all other routing protocols provided acceptable amount of jitter for video streaming. Finally, the simulation ran for five times, and the average obtained results are depicted in Figures 5, 6, 7, 8, 9, $10,11,12,13,14,15,16,17,18,19,20,21$ with $95 \%$ confidence interval $(\mathrm{CI})$. In the following figures, the 'hybrid' word refers to the GREENIE. End-to-end delay in all figures is measured in seconds. The results show that the proposed hybrid routing protocol (GREENIE) considerably outperforms other routing protocols, even if the network consists of several static, mobile mesh, and STA nodes.

Before discussing the obtained results, it is necessary to mention that a locator keeps the position of nodes in the designed hybrid WMN. The main goal of this locator is to know which STA or mesh node is connected to which MR. Actually, two main methods exist for finding the location of a node in WMNs. The first method keeps the position of the node in the routing table, and the second approach uses a locator. In the second case, entries in the routing tables are related only to the MRs, and the locator tables keep information about the location of nodes. A MR floods a packet to notify other MRs 
Table 2 Considered conditions and parameters in the simulation

\begin{tabular}{|c|c|c|c|}
\hline Parameter & Value(s) & Parameter & Value(s) \\
\hline Simulation time & $3,000 \mathrm{~s}$ & Number of STA nodes & $9,13,18,23,30$ \\
\hline Node distribution model & Uniform 20, 35 & Number of mesh nodes & 30 \\
\hline Mobility speed of mesh nodes & $\begin{array}{l}\text { Uniform } 5 \text { to } 20 \\
\text { mps }\end{array}$ & $\begin{array}{l}\text { Mobility speed of STA } \\
\text { nodes }\end{array}$ & Uniform 1 to $2 \mathrm{mps}$ \\
\hline Pause time in STA nodes & $\begin{array}{l}\text { Uniform } \\
100,500 \mathrm{~s}\end{array}$ & Packet size & 512 Bytes \\
\hline Transmission range & $140 \mathrm{~m}$ & Propagation model & Two ray \\
\hline Interference model & Additive & Hello interval in OLSR & $2 \mathrm{~s}$ \\
\hline TC interval in OLSR & $5 \mathrm{~s}$ & $\begin{array}{l}\text { OSLR willingness for all } \\
\text { nodes }\end{array}$ & 3 \\
\hline Network area size & $630 \times 1,000 \mathrm{~m}^{2}$ & Video stream type & CBR \\
\hline $\begin{array}{l}\text { Time between video packets } \\
\text { (per flow) }\end{array}$ & $0.08 \mathrm{~s}$ & $\begin{array}{l}\text { Confidence interval of } \\
\text { results }\end{array}$ & $95 \%$ \\
\hline Number of seeds in simulation & 5 & Route life time in DYMO & $10 \mathrm{~s}$ with link layer feedback \\
\hline Mobility model & $\begin{array}{l}\text { Random } \\
\text { waypoint }\end{array}$ & Binary bit rate & $54 \mathrm{Mbit} / \mathrm{s}$ \\
\hline MAC standard used & IEEE $802.11 \mathrm{~g}$ & \multirow{2}{*}{$\begin{array}{l}\text { Number of orthogonal } \\
\text { channels }\end{array}$} & \multirow{2}{*}{$\begin{array}{l}2 \text { (one used by the mesh network, one used between the STA } \\
\text { nodes and AP) }\end{array}$} \\
\hline Frequency & $2.4 \mathrm{GHz}$ & & \\
\hline Thermal noise & $-110 \mathrm{dBm}$ & $\begin{array}{l}\text { Maximum interference } \\
\text { range }\end{array}$ & $300 \mathrm{~m}$ \\
\hline Warm-up period & $100 \mathrm{~s}$ & Transmitter power & $2 \mathrm{mWatt}$ \\
\hline Routing protocols & & GREENIE (hybrid), & MP, proactive, reactive, spanning tree \\
\hline \multirow[t]{3}{*}{ Three simulation scenarios } & \multicolumn{3}{|c|}{ A. STA node request video stream from the server, mobile mesh nodes just relay the received packets } \\
\hline & \multicolumn{3}{|c|}{ B. STA nodes request video stream from the server, no mobile mesh node exists } \\
\hline & C. Thirty mesh $n$ & es exist in the network, but & $\begin{array}{l}\text { ine mobile mesh and nine STA nodes request video stream from } \\
\text { the server }\end{array}$ \\
\hline
\end{tabular}

about the new location of a node. In order to improve the efficiency of this locator, we modify its source code so that it can guarantee that the required information will be known to all nodes in the network. The locator module can be used to maintain the tables using two methods: the first is to send a flooding packet every time a change occurs in the locator tables (for example, due to a hand over of a node), whereas the locator can ask other locators in its neighbors for completely transferring their tables in the second method. Based on these methods and considered parameters in Table 2, the simulator ran for three scenarios. These scenarios cover all aspects of a

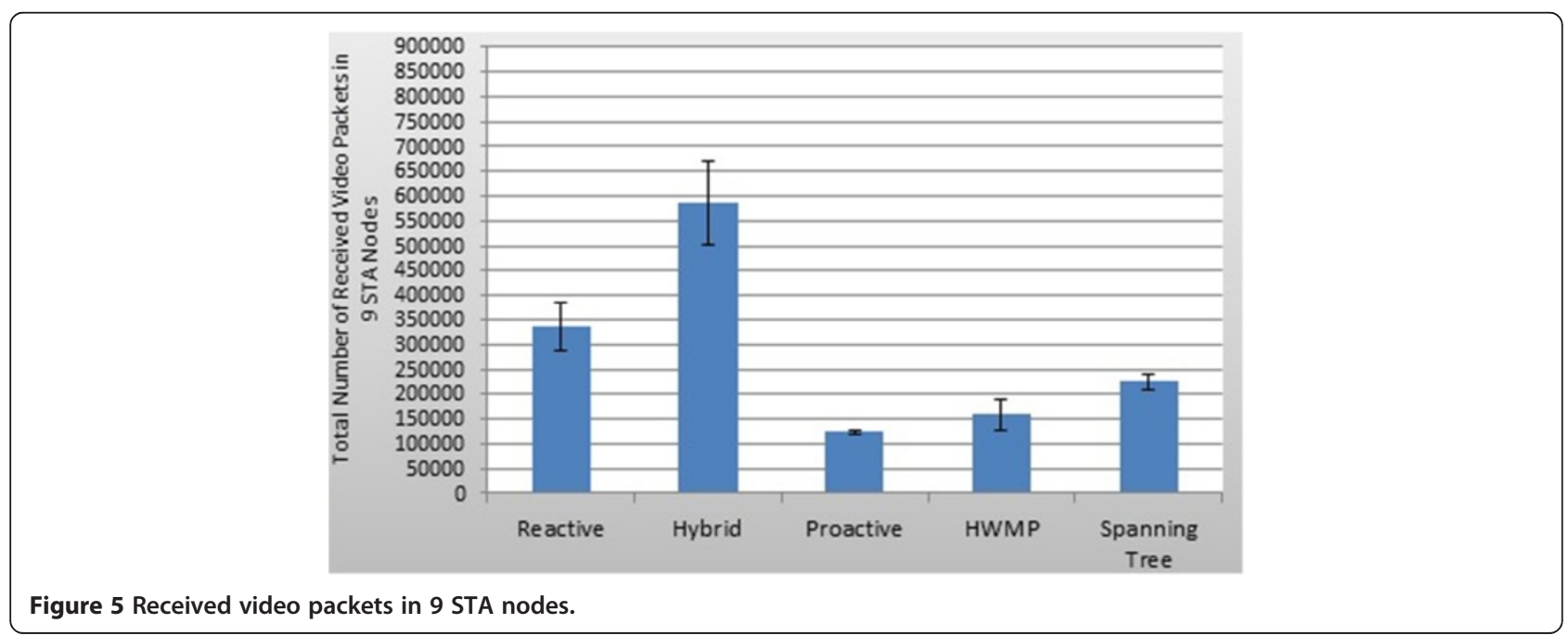




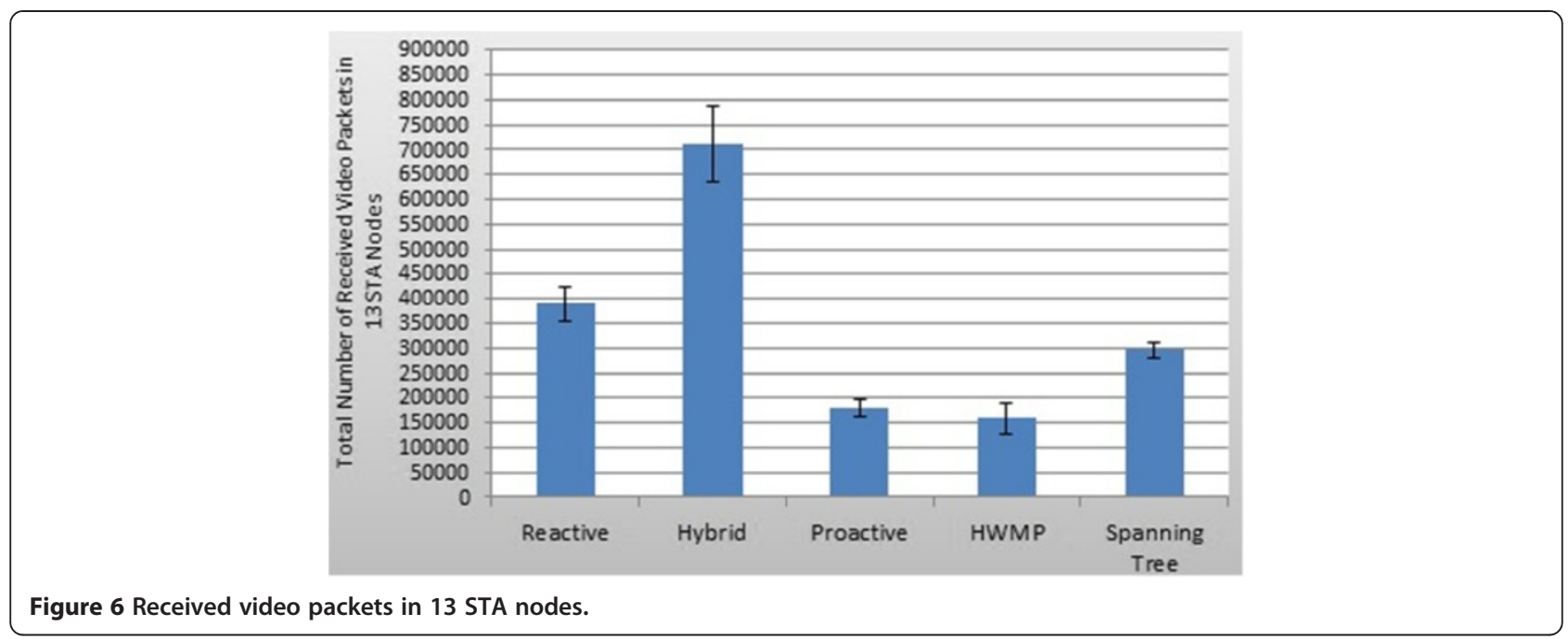

comprehensive evaluation precisely. In the following subsections, these scenarios and related obtained results are explained in great detail.

\subsection{STA nodes request the video stream from the video server, whereas mobile mesh nodes just relay the received packets (Scenario $A$ )}

In this scenario, all STA nodes request the video stream from the video server, and 30 mesh nodes just relay the received video packets to other nodes. Therefore, the number of nodes which playback the video stream equals the number of STA nodes. Figures 5, 6, 7, 8, 9 show the total number of successfully received video packets in a different number of STA node using different routing protocols. What can be inferred from the following figures is that GREENIE provides the highest performance in terms of the total number of successfully received packets in nodes.
Because of mobile mesh nodes, proactive routing protocol cannot provide considerable performance in comparison with others. In fact, nodes need to update their routing tables repeatedly, because links among them fail continually when they move on the network. Moreover, the reactive routing protocol is more robust in dynamic network including mobile nodes. However, using this type of routing protocols for the backbone is not suitable because MRs are fixed and using a reactive routing protocol increases the total required time for finding the best path between two MRs. Finally, both HWMP and spanning tree routing protocols are based on tree-based routing. Tree construction always increases the total required time for data dissemination and is not robust in high-node mobility and churning. Consequently, neither of these routing protocols can provide considerable performances in terms of the total number of successfully received packets.

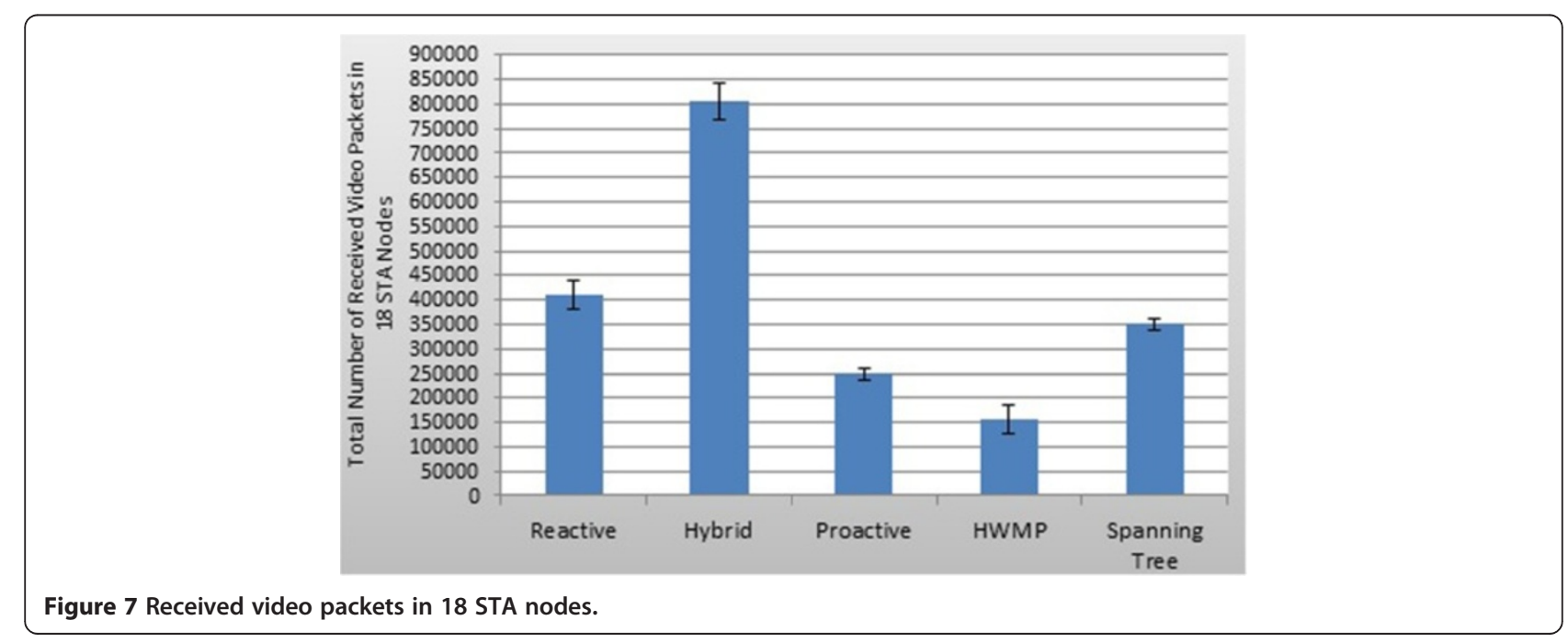




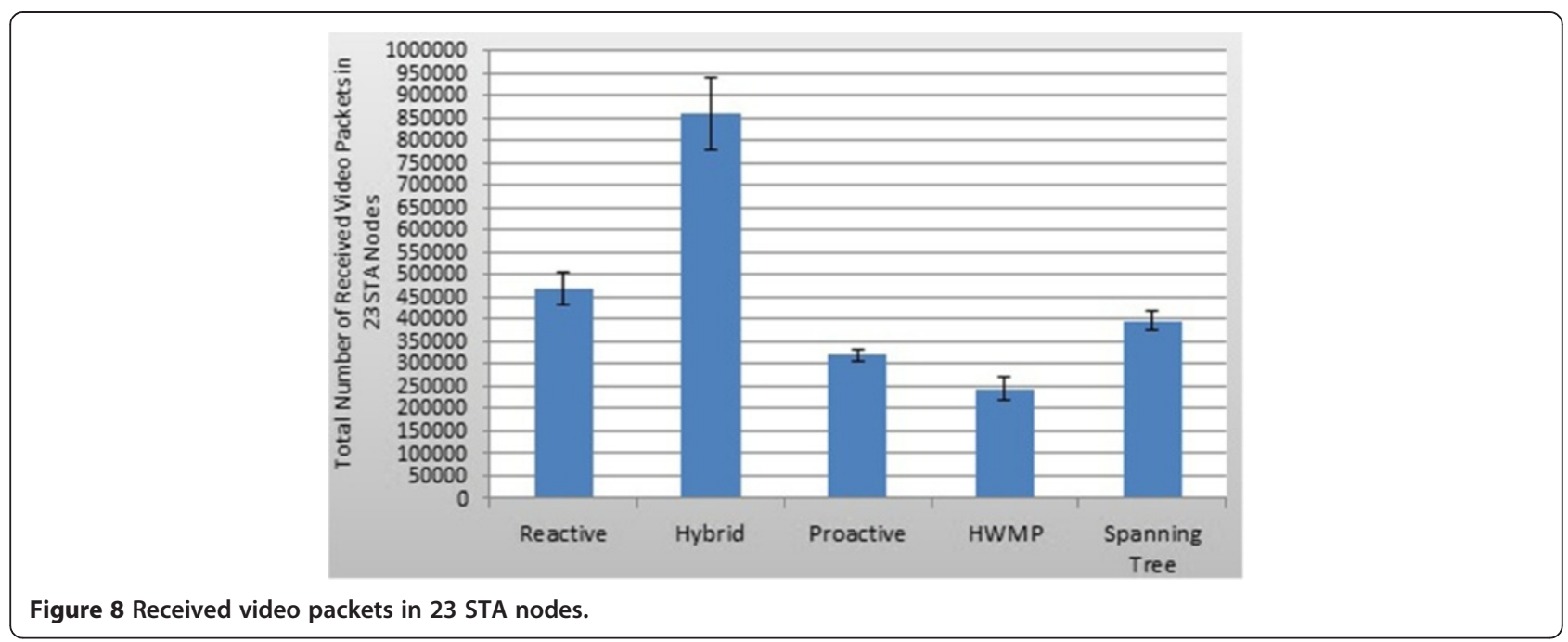

Figure 10 compares all examined routing protocols in this study for a different number of STA nodes. GREENIE lets STA nodes receive required video packets as many as possible. Like HWMP and reactive routing protocols, the behaviors of spanning tree and proactive routing protocols are the same to that when the number of STA nodes increases. The HWMP protocol is based on AODV which is a reactive routing scheme. Therefore, its behavior may look like the reactive protocols. Spanning tree protocol, like proactive scheme, finds the best path before starting the data transmission. Thus, it might look like the proactive protocol while the number of STA nodes increases. In addition to the total number of received video packets, this study measured the averaged value of end-to-end delay in STA nodes using different routing protocols as illustrated in Figures 11, 12, 13, 14, 15, 16. Contrary to our hybrid routing protocol, end-to-end delay did not remain constant in other routing protocols while the number of STA nodes increases. As it is expected, both the HWMP and the spanning tree protocols introduced higher end-to-end delays in comparison with other routing protocols; because they use tree-based structure for data transmission. However, the spanning tree method provides path redundancy which allows it to be more robust in dynamic networks including many mobile nodes. The HWMP protocol increases end-to-end delay moderately, whereas the introduced end-to-end delay by the spanning tree routing protocol is fluctuated for different number of nodes. According to previous studies, $20 \mathrm{~ms}$ is an acceptable value for the end-to-end delay in video streaming over WMNs. Hence, all routing protocols, except HWMP, could deliver video packets to applicant nodes before their playback time. This shows that an efficient hybrid routing protocol needs to consider many issues for providing considerable efficiency in data dissemination.

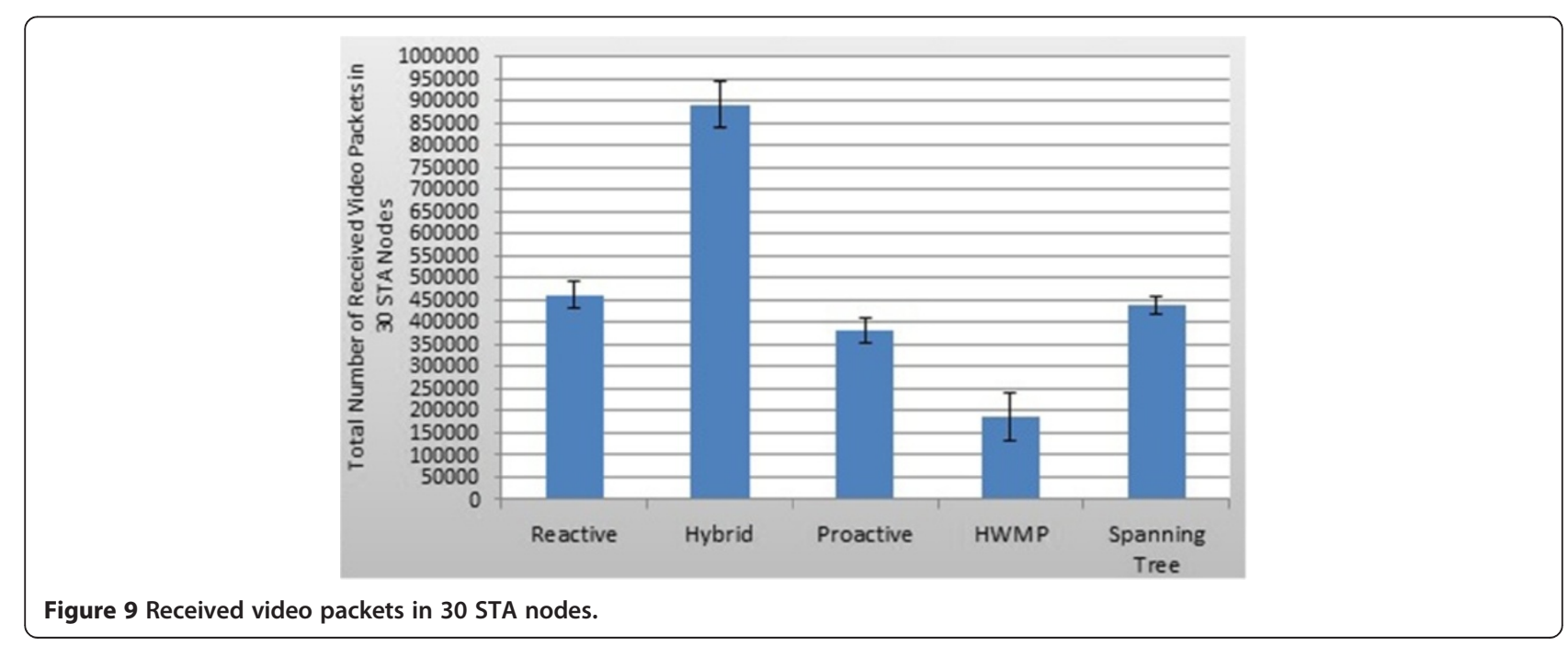




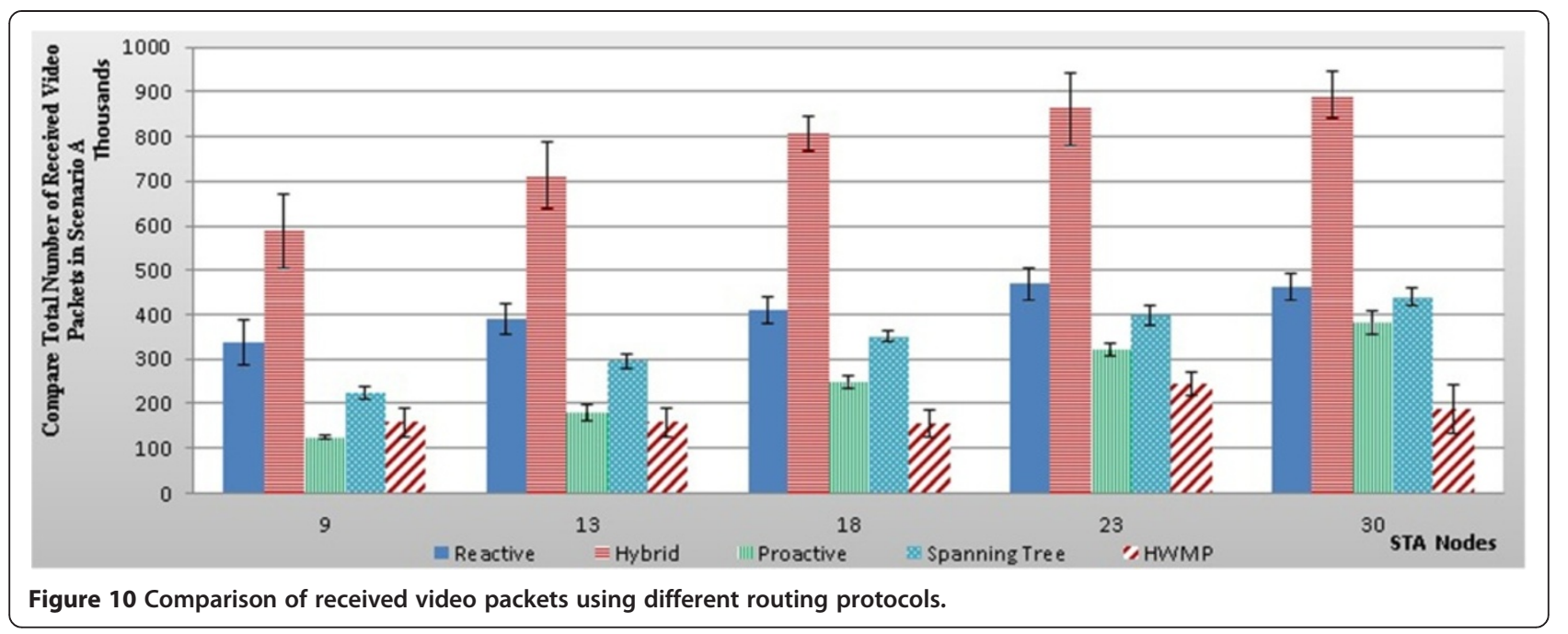

As illustrated in Figure 16, although the hybrid and the proactive schemes introduce low end-to-end delay in the system, the hybrid routing protocol provides, on the average, the least amount of end-to-end delay for different number of STA nodes without any fluctuation. In contrary to HWMP, the reactive routing protocol does not impose high end-to-end delay in the system because all packets use the same path and the initial cost of finding the path (the initial delay) is compensated by the other packets. However, the hybrid scheme considerably outperforms the reactive protocol. According to the obtained results by the HWMP, a hybrid scheme which is based on both reactive and proactive protocols cannot necessarily provide high performances. In other words, many different issues must be considered for designing and implementing an efficient hybrid method.
7.2. STA nodes request the video stream from the video server, but no mobile mesh node exists in the network (Scenario B)

In this scenario, different number of STA nodes is considered, but no mesh node exists in the network. It means that there is no mobile node with high mobility speed on the network. Figures 17, 18 show the total number of successfully received video packets and the end-to-end delay provided by different routing protocols for various numbers of STA nodes. As illustrated in Figure 17, GREENIE provides the best performance. Proactive routing protocol introduces the minimum end-to-end delay because it uses a predefined path in the routing tables and there is no mobile node in the network. However, it is not realistic to imagine a wireless network with no mobile nodes. Figures 17, 18 depict that the hybrid and proactive

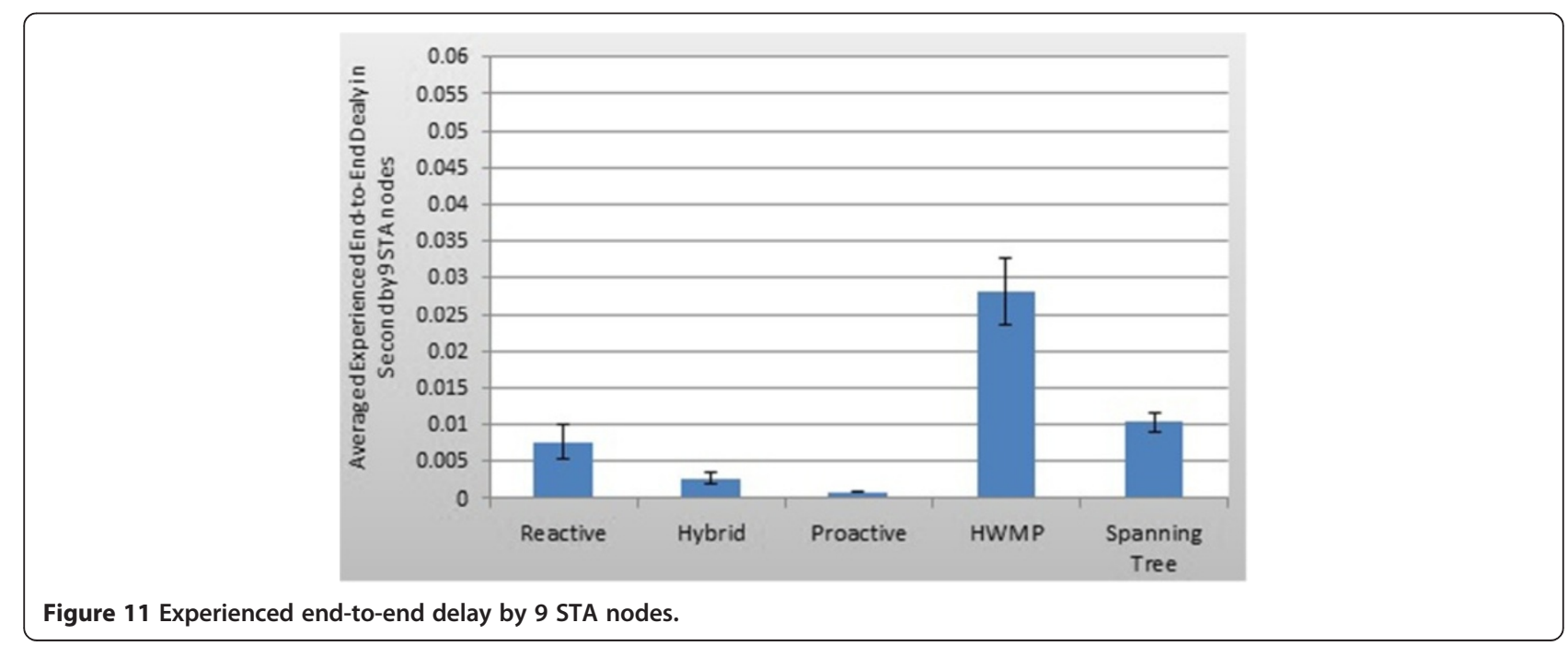




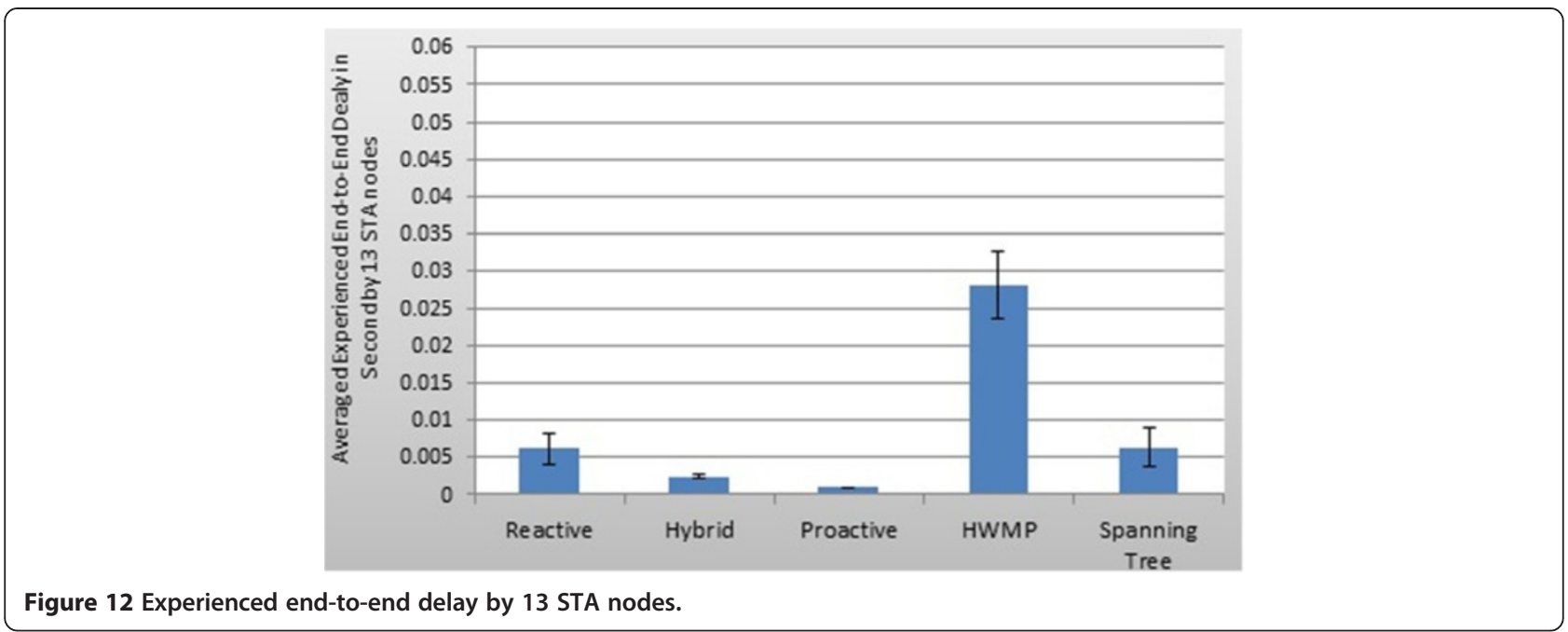

protocols provide approximately the same performances in terms of the total number of successfully received video packets and the end-to-end delay in non-dynamic network. This means that the proposed hybrid routing protocol, contrary to the HWMP, efficiently exploits the benefits of proactive protocols in static network.

\subsection{Thirty mesh nodes exist in the network, but nine} mobile mesh and nine STA nodes request the video stream from the video server (Scenario $\mathrm{C}$ )

In the last scenario, 9 STA and 30 mesh nodes exist in the network, but just 9 mesh nodes request video stream from the server. Figures 19, 20 depict the obtained results for the averaged total number of successfully received video packets and the experienced end-to-end delay in nine STA and mesh nodes, respectively. According to the following figures, the hybrid scheme provides the best performance and introduces low end-to-end delay similar to proactive routing protocol.

\subsection{Routing overhead}

Finally, this section shows the imposed routing overhead by each of the considered routing protocols in this study. The results are depicted in Figure 21. This figure shows the normalized values of routing overhead for different numbers of STA nodes. It is necessary to mention that all thirty mesh nodes participated in the routing. The hybrid routing protocol shows approximately fixed routing overhead with low fluctuation for different number of STA nodes. The HWMP provides the least routing overhead when the number of STA nodes exceeds 18 . In this experiment, most of the routing packets are lost by collisions. Therefore, those routing protocols such as HWMP which send fewer routing packets provide better performances in terms of routing overhead when there is

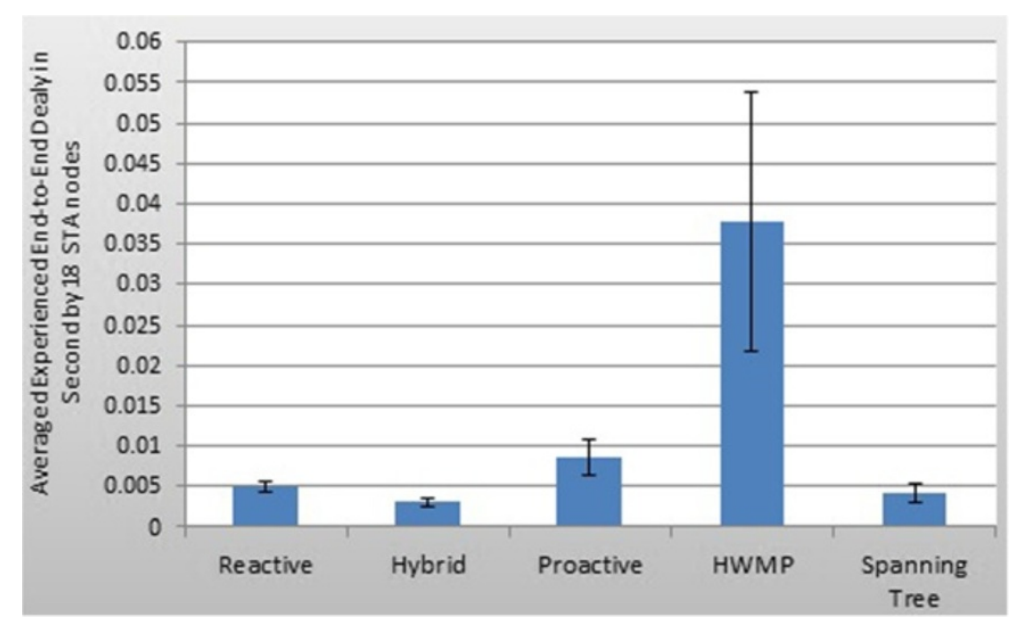

Figure 13 Experienced end-to-end delay by 18 STA nodes. 


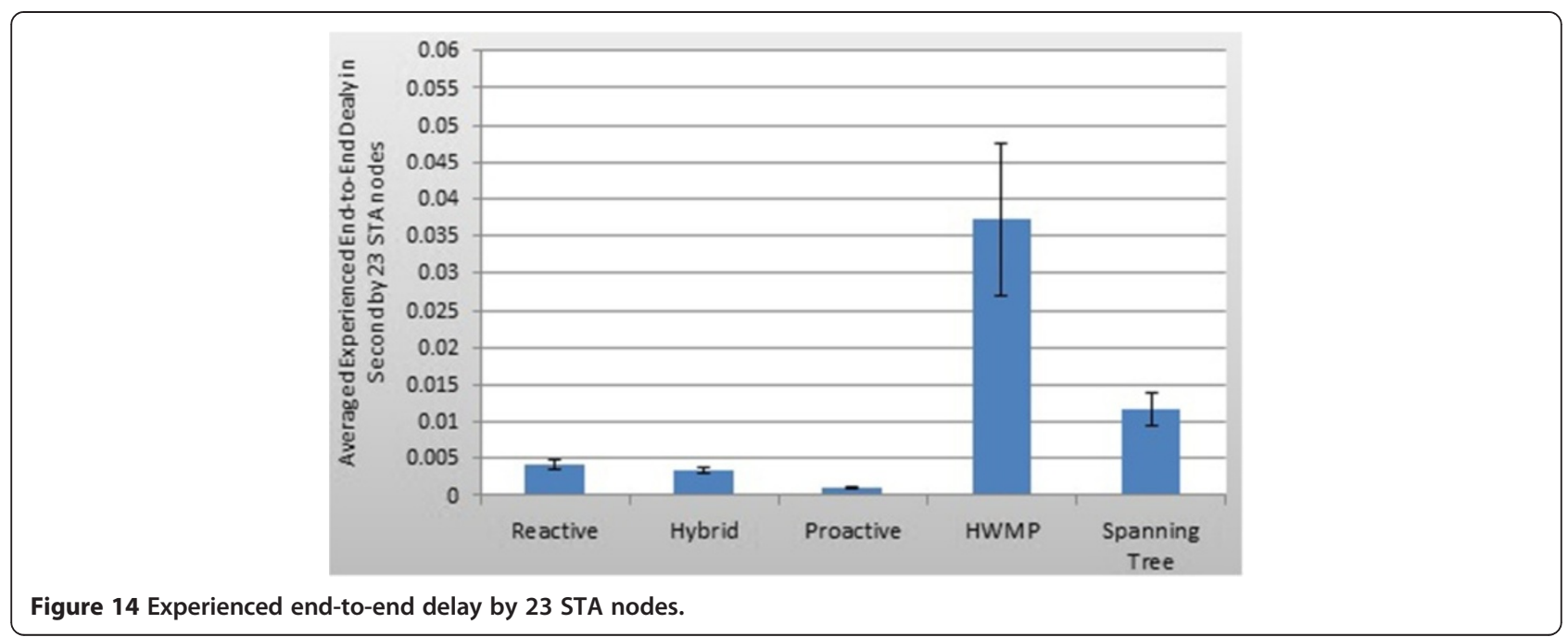

a large number of nodes in the network. In addition, the imposed routing overhead by proactive routing protocol decreases when the number of nodes increases. However, if the number of mobile nodes increases in the network, these types of routing protocols will suffer from high routing overhead, because they continually need to find new routing paths. As a result, because of a low introduced overhead to the network, GREENIE (hybrid) is more reliable for providing smooth video playback on receivers. We can also conclude that the use of hybrid routing protocols reduce the energy consumption as a lower number of messages are introduced into the network.

\section{Remaining issues and future works}

As mentioned in the previous sections, many recent studies have introduced or improved the efficiencies of WMNs for data dissemination. Altogether, there are some important remaining challenges and issues which can be classified as follows:

1. A trade-off between the cost of the network and the number of routers and interfaces.

2. New solution for the best integration between WMN and other wireless standards in terms of cost and performance.

3. More efficient methods for decreasing the complexity and the cost of some current systems such as multiinput multi-output, smart antenna, direct antenna, and frequency agile/cognitive in WMNs.

4. More efficient solutions for providing the necessary level of QoS regarding different types of traffics.

5. Improve the existing or introduce the new solutions for providing required advanced MAC, network, and physical layer techniques for better mobility capability with nodes.

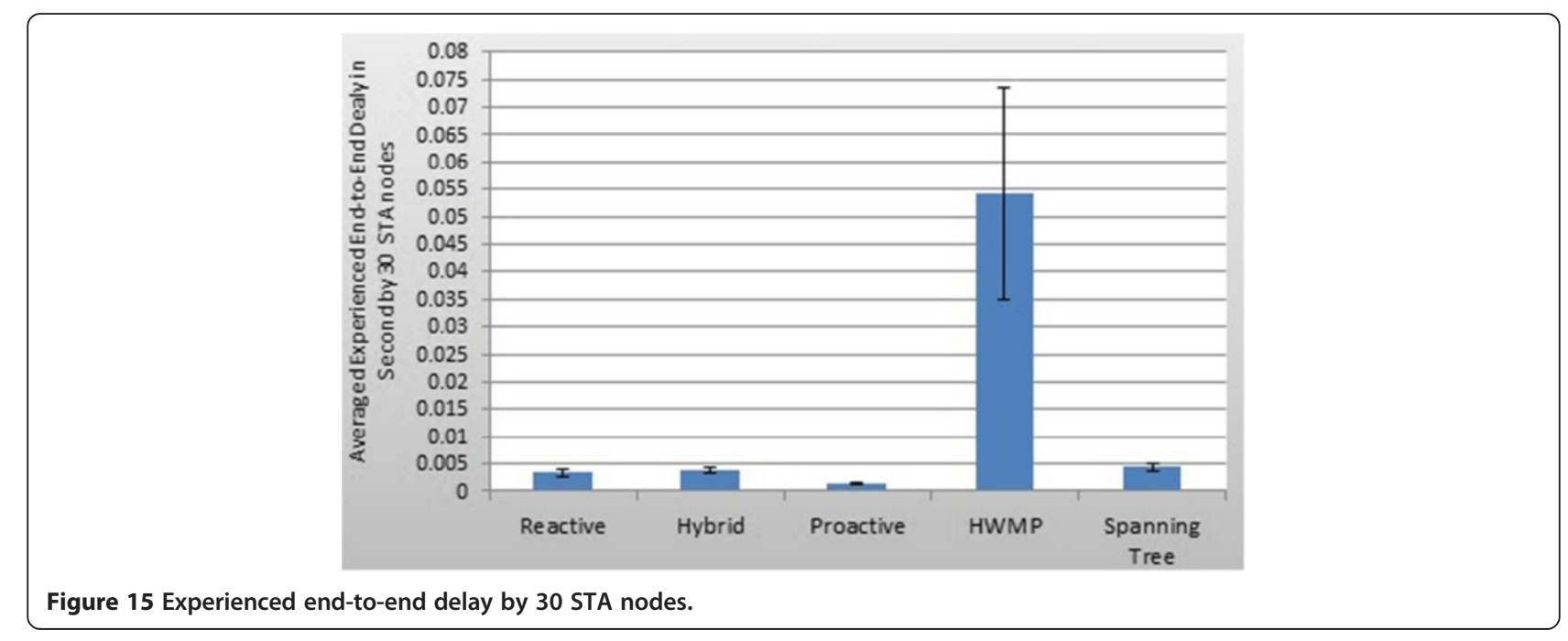




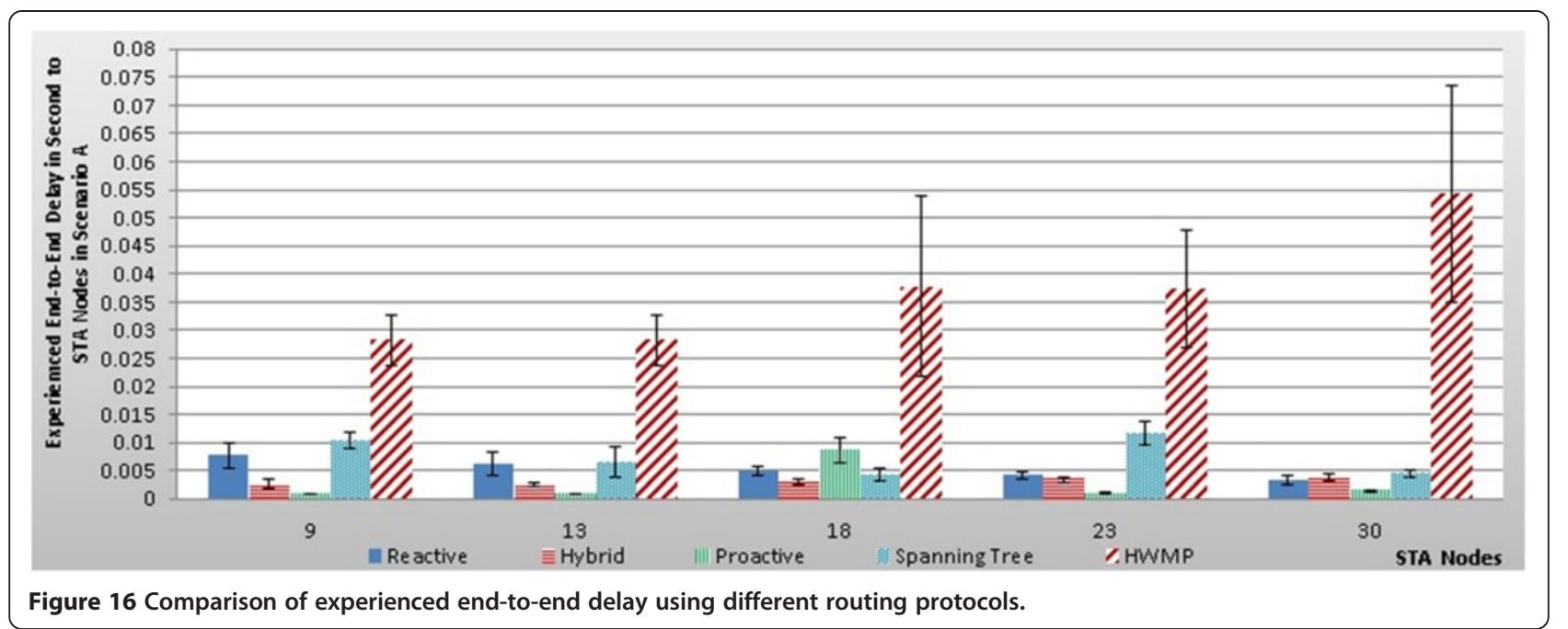

6. Improve the existing or introduce new highperformance algorithms and protocols to cope with security issues in WMNs. Although some recent studies, such as [65] focus on internal attacks due to compromised nodes, the necessity of introducing more efficient algorithms is inevitable.

7. More efficient link metrics are required so that they consider the peculiarities of multi-channel multiradio WMNs.

8. The interactive integration of routing and MAC layer can help maximize their performances.

9. One of the most important open issues in the MAC layer is that a node can access multiple physical rates. In other words, the capacity of the link decreases when the physical rate reduces.

Different data stream types require different exchange policies. Therefore, it is necessary to evaluate and select routing protocols and metrics which consider these factors completely and precisely. Moreover, some other aspects such as density, coverage area, number of nodes, and the number of mobile nodes need to be considered. For example, proactive routing protocols are more efficient for delay sensitive streams. However, they cannot provide good performance in high mobility rate. In addition to selecting a suitable routing protocol, considering effective routing metrics is really important. For example, when the route between two nodes changes frequently due to the selected metric, the performance of the network degrades sharply. Dynamic metrics provide higher performance, when the number of flows is small and the probability of interference is low.

However, if the network is overloaded, the dynamic metric usually is inefficient. In this case, a simple minimum hop count metric provides better performance than that of dynamic metrics. Consequently, different metrics for different overload situations should be considered. Although using the same metrics at the same time can be an

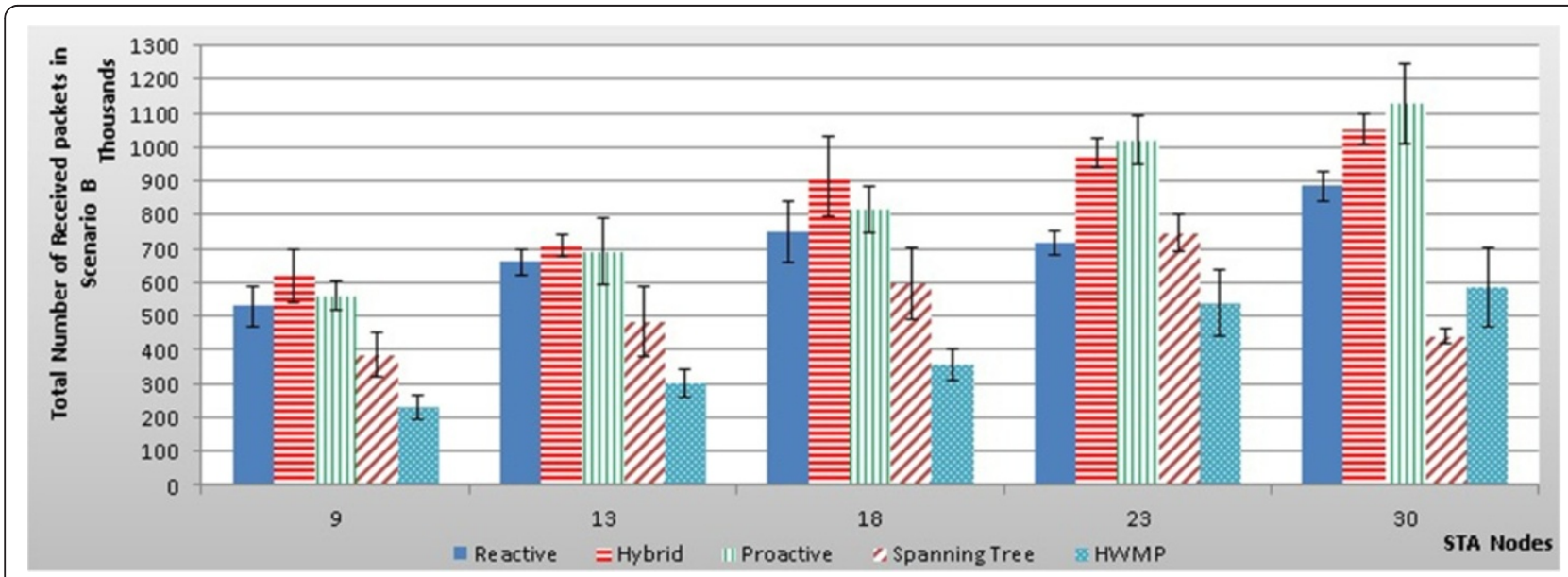

Figure 17 Averaged number of successfully received packets in scenario B. 


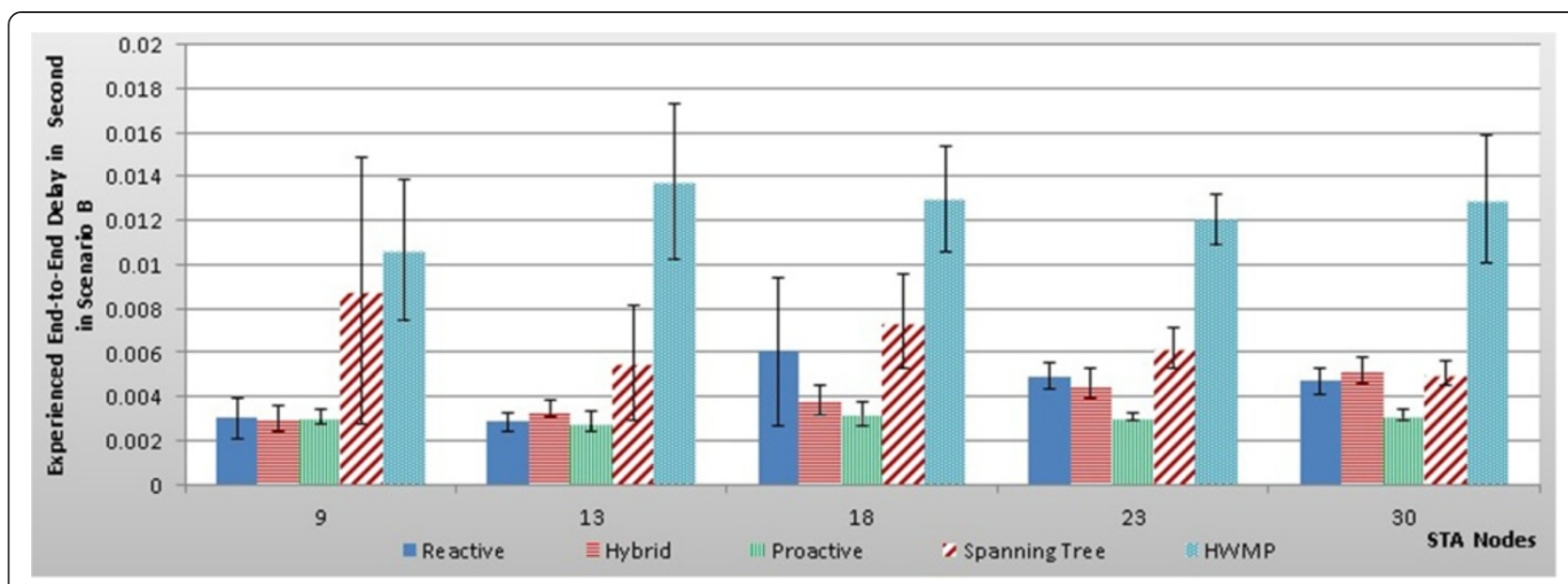

Figure 18 Averaged experienced end-to-end delay in scenario B.

easy solution, it imposes high complexity to the network. Thus, it is better to have different metrics in function of the status of the network. All in all, different routing protocols and metrics provide different levels of performances. These important points warn us that more research should be performed on the existing routing protocols and metrics in order to provide efficient data dissemination among wireless nodes, even if there are many mobile nodes in the network. Some recent studies such as [66] and [67] considered two other challenges including the required level of QoS and performance in a WMN, respectively.

\section{Conclusions}

Among all existing applications, video streaming causes a large portion of traffics in computer network, especially in the Internet. Moreover, users are really interested in joining wireless networks. Among all wireless network standards, WMNs provide the best scalable infrastructure for end users. Therefore, video streaming over WMNs has become very popular in recent years. However, there are some challenges in providing smooth video playback in this type of networks. For example, mobility, noise, interference, contention for available free channels and limited available bandwidth are some important problems, which need to be considered. Efficient routing protocols can reduce the side effects of these problems on the perceived video quality. Hence, it is important to completely understand the efficiencies of these routing protocols for video streaming, when mobile mesh nodes exist in the WMN. The present study introduced an efficient hybrid routing protocol and compared it with the most important recently used routing protocols in WMNs for video streaming using OMNET++ simulator. This study considered three scenarios for simulation:

In the first scenario, STA node request video stream from the server, but mobile mesh nodes just relay the received packets. In the second scenario, STA nodes request video stream from the server and no mobile mesh node exists in the network. Finally, in the third scenario,

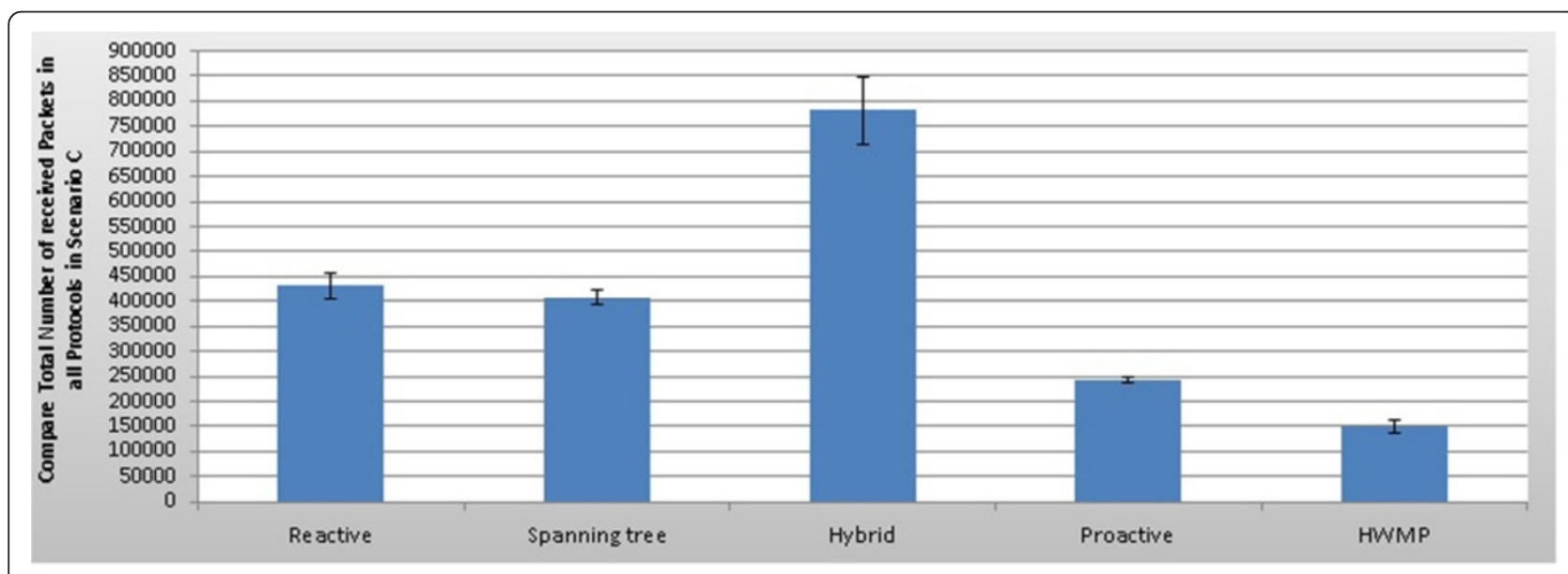

Figure 19 Averaged number of successfully received packets in scenario C. 


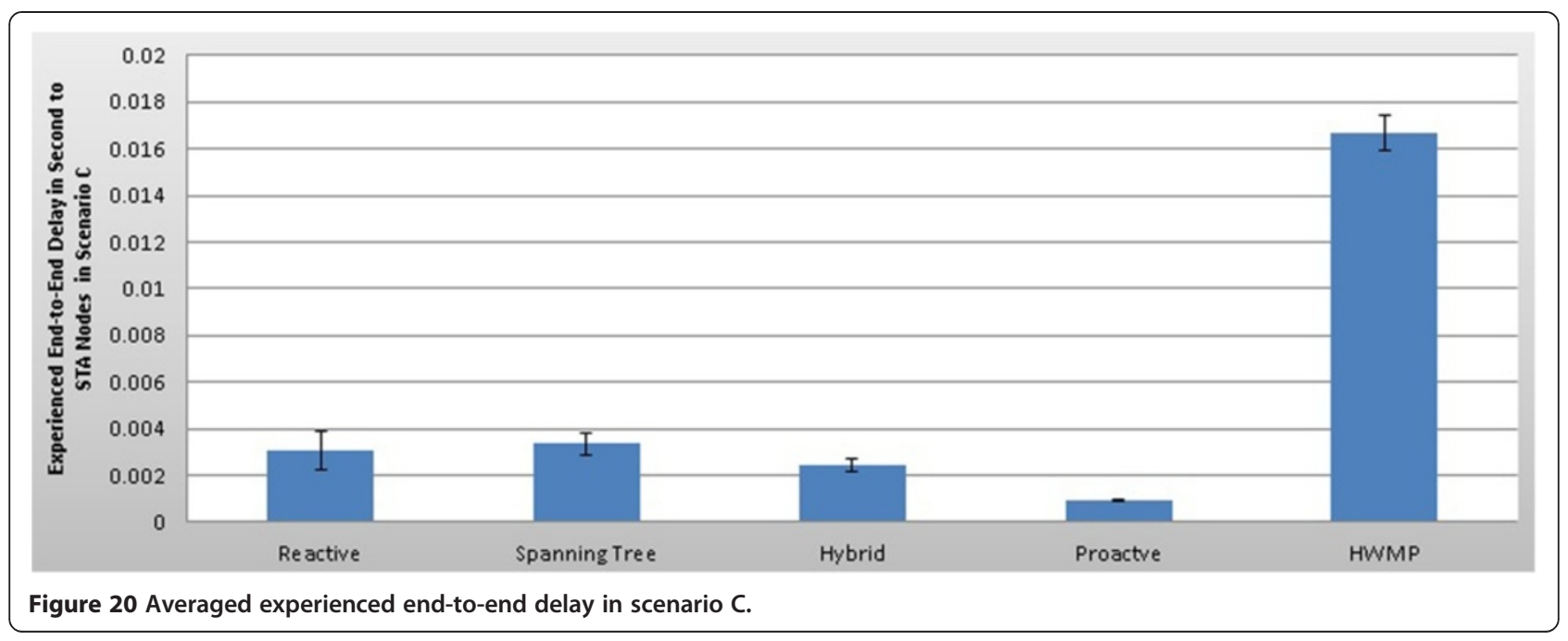

nine mobile mesh and nine STA nodes request video stream from the server where other 21 mobile mesh nodes just relay the received video packets. The obtained results for the total number of successfully received packets, end-to-end delay, and routing overhead are depicted in figures with $95 \%$ confidence interval. The results show that the new proposed hybrid routing protocol outperforms others and provides higher video quality on receivers. In the future work, we will examine the efficiencies of these routing protocols in real testbed. Moreover, this study will consider a modified locator module so that some nodes can be awarded about the new location of a mobile node and some of them remain unaware.

\section{Methods}

This study compared the efficiencies of different routing protocols in video streaming and compared them with the proposed hybrid scheme. This study considered three scenarios. The first scenario includes both STA and mobile mesh nodes, but just STA nodes request the video

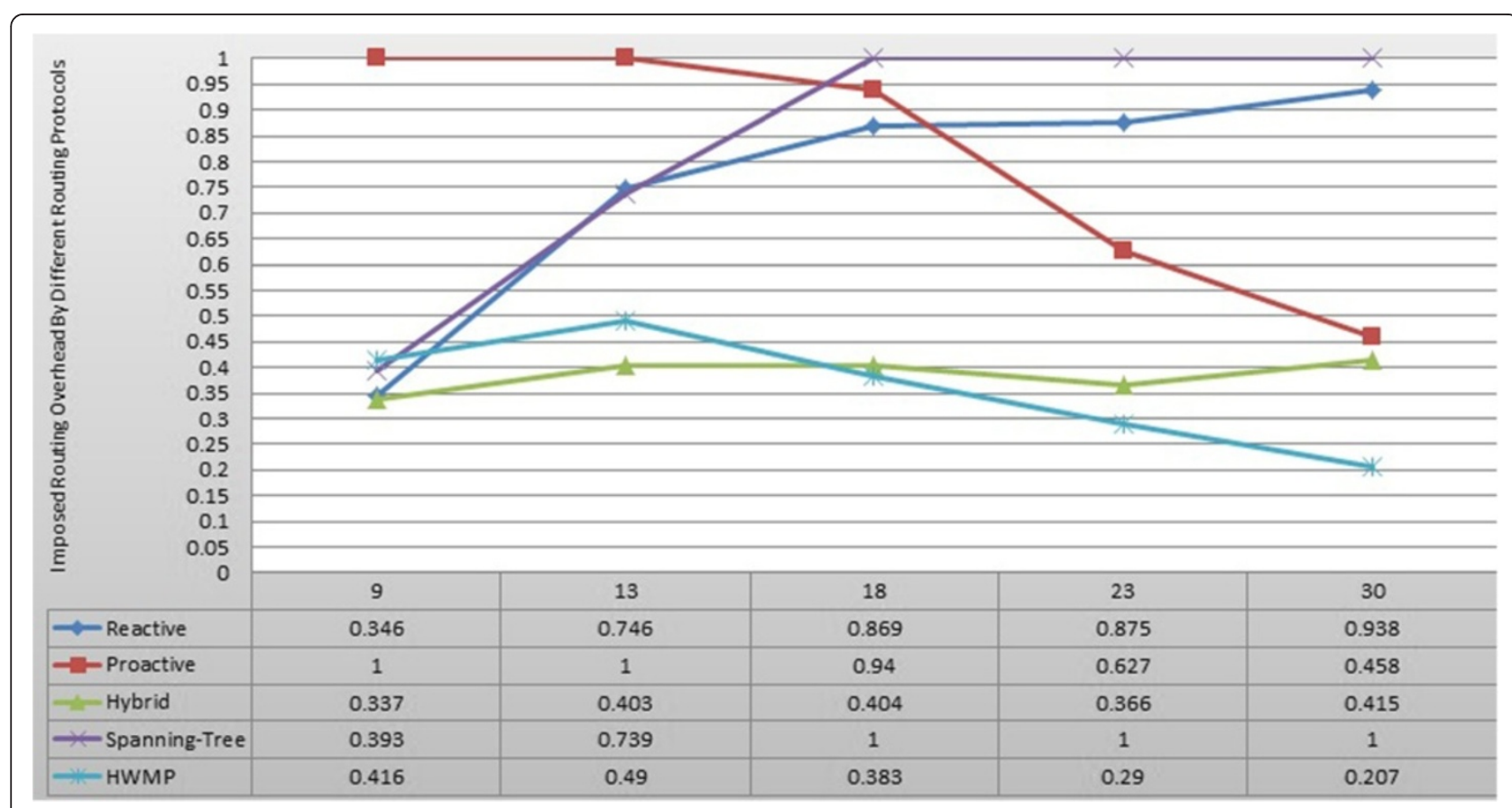

Figure 21 A comparison among imposed routing overhead by each routing protocol. 
traffic and mesh nodes just relay received video packets. In the second scenario, only STA nodes are considered and the third scenario includes both STA and mesh nodes such that some of mesh nodes request the video traffic. A video source disseminated the video stream in all scenarios. This video stream is encoded using CBR technique. Three important performance metrics including the total number of successfully received packets, end-to-end delay, and routing overhead are measured for different routing protocols.

\section{Notes}

This work compares important existing routing protocols with the proposed hybrid protocol in different scenarios. Video streaming will be the most important type of traffics over computer networks in the near future. Moreover, WMNs provide efficient infrastructure for user with regard to their provided benefits. Therefore, video streaming over WMNs will be one of the most attractive applications in the near future. The results of this work help other researchers to understand the efficiency of existing routing protocols in WMNs for video streaming.

\begin{abstract}
Abbreviations
AVC: Advance video coding; AODV: Ad-hoc on-demand distance vector; CBR: Constant bit rate; Cl: Confidence interval (95\% in this study); EED: Endto-end delay; GoP: Group-of-pictures; HWMP: Hybrid wireless mesh protocol; MANET: Mobile ad-hoc network; MP: Mesh point; MAP: Mesh access point MPP: Mesh portal point; MR: Mesh router; MAC: Medium access control; P2P: Peer-to-peer; QoS: Quality-of-service; RREP: Route reply; RREQ: Route request; SVC: Scalable video coding; UAV: Unmanned aerial vehicles; VBR: Variable bit rate; VoD: Video-on-demand; WMN: Wireless mesh network.
\end{abstract}

\section{Competing interests}

The authors declare that they have no competing interests.

\section{Acknowledgements}

This research is supported by the Research Management Center (RMC) Universiti Teknologi Malaysia (UTM) using the Prototype Research Grant Scheme (PRGS) from the Ministry of Higher Education (MOHE) using vote number R.J130000.7828.4 L600.

\section{Author details}

${ }^{1}$ Faculty of Computing, Universiti Teknologi Malaysia, Johor Bahru 81310, Malaysia. ${ }^{2}$ Dpto Tecnología Electrónica, E.T.S.I, Telecommunication, University of Malaga, Malaga 29071, Spain. ${ }^{3}$ Escuela de Ingenierías, Dpto. Ingeniería Eléctrica, University of Malaga, Malaga 29071, Spain.

Received: 10 October 2012 Accepted: 16 May 2013

Published: 18 June 2013

\section{References}

1. GRHD Denteneer, S Max, R Taori, J Cardona, L Berlemann, B Walke, IEEE 802.11s: the WLAN mesh standard. Wirel. Commun. IEEE 17(1), 104-111 (2010)

2. Y Zhang, J Luo, H Hu, Wireless Mesh Networking: Architectures, Protocols and Standards (Wireless Networks and Mobile Communications) (Auerbach Publications, Boca Raton, 2007)

3. S Ahmed, MS Alam, Performance evaluation of important ad hoc network protocols. EURASIP J. Wirel. Commun. Netw. 2006(2), 42-42 (2006)

4. IEEE, 802.11s-2011 - IEEE Standard for Information TechnologyTelecommunications and information exchange between systems-Local and metropolitan area networks-Specific requirements Part 11: Wireless LAN
Medium Access Control (MAC) and Physical Layer (PHY) specifications Amendment 10: Mesh Networking (IEEE, Orlando, 2011)

5. IF Akyildiz, X Wang, W Wang, Wireless mesh networks: a survey. Comp. Netw. 47(4), 445-487 (2005)

6. M Asri Bin Ngadi, S Ali, A Hanan Abdullah, R Hafeez Khokhar, A taxonomy of cross layer routing metrics for wireless mesh networks. EURASIP J. Wirel. Commun. Netw. 177 (2012)

7. LF Akyildiz, X Wang, Wireless Mesh Networks (Wiley, New York, 2009)

8. R Karrer, A Pescape, T Huehn, Challenges in second-generation wireless mesh networks. EURASIP J. Wirel. Commun. Netw. 2008, 274790 (2008)

9. M Sichitiu, Wireless mesh networks: opportunities and challenges. EURASIP J. Wirel. Commun. Netw. , 318-323 (2005)

10. KN Ramachandran, M Buddhikot, G Chandranmenon, S Miller, E BeldingRoyer, K Almeroth, On the design and implementation of infrastructure mesh networks, in Proceedings of the IEEE Workshop on Wireless Mesh Networks (WiMesh). Santa Clara,2005 (IEEE, Orlando, 2005)

11. S Kumar, Reactive and Proactive Routing Protocols for Wireless Mesh Network using Multimedia Streaming[A], in Proceedings of the International Conference on Recent Advances and Future Trends in Information Technology (iRAFIT 2012) (International Journal of Computer Applications, Special Issue[C], 2012)

12. B Barekatain, MA Maarof, Network coding efficiency in live video streaming over Peer-to-Peer Mesh networks, in The 7th International Conference on Information Technology in Asia (CITA 11), Kuching, 12-13 July 2011 (IEEE, Orlando, 2011), pp. 1-7

13. IE Richardson, The H.264 advanced video compression standard (Wiley, New York, 2010)

14. S Montazeri, M Fathy, R Berangi, An adaptive fair-distributed scheduling algorithm to guarantee QoS for both VBR and CBR video traffics on IEEE 802.11e WLANs. EURASIP J. Adv. Signal Proc. 2(8), 1-12 (2008)

15. J Maatta, T Braysy, A novel approach to fair routing in wireless mesh networks. EURASIP J. Wirel. Commun. Netw. 2009, 1-13 (2009)

16. MEM Campista, PM Esposito, IM Moraes, LHMK Costa, OCMB Duarte, DG Passos, CVN de Albuquerque, DCM Saade, MG Rubinstein, Routing metrics and protocols for wireless mesh networks. Netw. IEEE 22(1), 6-12 (2008)

17. A Capone, G Carello, I Filippini, S Gualandi, F Malucelli, Routing, scheduling and channel assignment in wireless mesh networks: optimization models and algorithms. Ad Hoc Netw. 8(6), 545-563 (2010)

18. G Held, Wireless Mesh Networks led (Auerbach Publications, New York, 2005)

19. L Sanghwan, Y Yu, S Nelakuditi, Z-L Zhi, C-N Chuah, Proactive vs reactive approaches to failure resilient routing, in INFOCOM 2004. Twenty-third AnnualJoint Conference of the IEEE Computer and Communications Societies, Hong Kong, March 2004 vol. 1 (IEEE, Orlando, 2004), p. 186. 7-11

20. CE Perkins, P Bhagwat, Highly dynamic destination-sequenced distancevector routing (DSDV) for mobile computers. SIGCOMM Comput. Commun. Rev. 24(4), 234-244 (1994)

21. X Qiu, H Liu, D Ghosal, B Mukherjee, J Benko, W Li, R Bajaj, Enhancing the performance of video streaming in wireless mesh networks. Wirel. Pers. Commun. 56(3), 535-557 (2011)

22. P Jacquet, Optimized link state routing protocol for ad hoc networks, in IEEE INMIC 2001. Technology for the 21st Century Proceedings (IEEE International, France, 2001), pp. 62-68

23. DB Johnson, DA Maltz, Dynamic Source Routing in Ad Hoc Wireless Networks, in Mobile Computing, ed. by T Imielinski, H Korth. Chapter 5 (Kluwer Academic Publishers, Aarhus, 1996), pp. 153-181

24. EM Royer, CE Perkins, An implementation study of the AODV routing protocol, in IEEE Wireless Communications and Networking Conference, 2000. WCNC. Chicago, 2000, vol. 3 (IEEE, Orlando, 2000), pp. 1003-1008

25. C-K Toh, Associativity-based routing for ad hoc mobile networks. Wirel. Pers. Commun. 4(2), 103-139 (1997)

26. HLH Jun, Y Feng, A noval hybrid wireless routing protocol for WMNs, in 2010 International Conference On Electronics and Information Engineering (ICEIE), Kyoto, 1-3 August 2010, vol.1 (IEEE, Orlando, 2010)

27. M Abolhasan, T Wysocki, E Dutkiewicz, A review of routing protocols for mobile ad hoc networks. Ad Hoc Netw. 2(1), 1-22 (2004)

28. M Bahr, Update on the hybrid wireless mesh protocol of IEEE $802.11 \mathrm{~s}$, in IEEE International Conference on Mobile Ad hoc and Sensor Systems, MASS 2007. Pisa, 8-11 Oct. 2007 (IEEE, Orlando, 2007), pp. 1-6

29. X Hong, Scalable routing protocols for mobile ad hoc networks. Netw. IEEE 16(4), 10 (2002)

30. IF Akyildiz, W Xudong, A survey on wireless mesh networks. Commun. Mag. IEEE 43(9), S23-S30 (2005) 
31. T Tsai, S Tsai, A cross-layer routing design for multi-interface wireless mesh networks. EURASIP J. Wirel. Commun. Netw. 4, 1-4 (2009). 8

32. C Wei, X Sun, Y Li, Research on QoS Routing Protocols, in Wireless Mesh Networks Frontiers in Computer Education, ed. by S Sambath, E Zhu (Springer Berlin, Heidelberg, 2012), pp. 1165-1172

33. KUR Khan, RU Zaman, AV Reddy, Performance comparison of on-demand and table driven ad hoc routing protocols using NCTUNs, in The Tenth International Conference on Computer Modeling and Simulation, UKSIM 2008. 1-3 April 2008, 2008, pp. 336-341

34. NS Mohamad, UA Abdullah, Performance Evaluation of AODV, DSDV \& DSR Routing Protocol in Grid Environment. IJCSNS Intern. J. Comput. Sci. Netw. Secur. 9, 7 (2009)

35. MA Rahman, MS Islam, A Talevski, Performance measurement of various routing protocols in ad-hoc network, in Proceedings of the International Multi Conference of Engineers and Computer Scientists IMECS 2009, Hong Kong, March, 2009, Vol 1, 2009, pp. 18-20

36. A Zakrzewska, L Koszalka, I Pozniak-Koszalka, A Kasprzak, International Conference on Computational Science and Its Applications (ICCSA), Fukuoka, March 2010 Analysis of Routing Protocol Performance in Wireless Mesh Networks (IEEE, New York, 2010), pp. 307-310

37. K Nenad, R Irini, R Branimir, A neural networks-based hybrid routing protocol for wireless mesh networks. Sensors 12(6), 7548-7575 (2012)

38. S Sumathi, Computational Intelligence Paradigms: Theory \& Applications using MATLAB (Taylor \& Francis Group, LLC, Boca Raton, 2010)

39. K Ramachandran, M Buddhikot, G Chandranmenon, S Miller, E BeldingRoyer, K Almeroth, Proceedings of the IEEE WiMesh, Santa Clara, September 2005 On the design and implementation of infrastructure mesh networks (IEEE, New York, 2005)

40. A-N Le, D-W Kum, Y-Z Cho, An Efficient Hybrid Routing Approach for Hybrid Wireless Mesh Networks, in Advances in Information Security and Assurance, Lecture Notes in Computer Science,vol. 5576, ed. by J Park, H-H Chen, M Atiquzzaman, C Lee, K T-h, S-S Yeo (Springer, Berlin Heidelberg, 2009), pp. 532-542

41. Y Amir, C Danilov, R Musaloiu-Elefteri, N Rivera, An inter-domain routing protocol for multi-homed wireless mesh networks, in IEEE International Symposium on a World of Wireless, Mobile and Multimedia Networks, WoWMoM 2007. Espoo, 18-21 June 2007 (IEEE, Orlando, 2007), pp. 1-10

42. N Peppas, D Turgut, A Hybrid Routing Protocol in Wireless Mesh Networks, in Military Communications Conference, 2007. MILCOM 2007 (IEEE, Orlando, 2007), pp. 1-7

43. S Mir, AA Pirzada, M Portmann, HOVER: hybrid on-demand distance vector routing for wireless mesh networks, in Proceedings of the thirty-first Australasian conference on Computer science - Volume 74 (Australian Computer Society, Inc, Wollongong, Australia, 2008), pp. 63-71

44. Z Zhang, RW Pazzi, A Boukerche, A mobility management scheme for wireless mesh networks based on a hybrid routing protocol. Comput. Netw. 54(4), 558-572 (2010)

45. X Cheng, P Mohapatra, S Lee, S Banerjee, Performance evaluation of video streaming in multihop wireless mesh networks, in Proceedings of the 18th International Workshop on Network and Operating Systems Support for Digital Audio and Video (ACM, Braunschweig, Germany, 2008), pp. 57-62

46. A Rajanikanth, B Nitin, B Supriya, Efficient real-time video transmission in wireless mesh network. Int. J. Res. Comput. Sc. 2(1), 11-19 (2011)

47. FR Dogar, A Phanishayee, H Pucha, O Ruwase, D Andersen, Ditto: a system for opportunistic caching in multi-hop wireless networks, in Proceedings of the 14th ACM International conference on Mobile computing and networking, San Francisco, September 2008 (ACM, New York, 2008), pp. 279-290

48. L Yinan, C Ing-Ray, APPCCM: Adaptive per-user per-object cache consistency management for mobile client-server applications in wireless mesh networks, in 2010 IEEE 35th Conference of the Local Computer Networks (LCN), Denver, October 2010 (IEEE, New York, 2010), pp. 128-135

49. Z Yingnan, Z Wenjun, L Hang, G Yang, S Mathur, Supporting video streaming services in infrastructure wireless mesh networks: architecture and protocols, in IEEE International Conference on Communications, ICC '08. Beijing, 19-23 May 2008 (IEEE, Orlando, 2008), pp. 1850-1855

50. S Nichols, KA Hua, Design and implementation of intelligent mesh nodes for wireless video stream sharing, in Proceedings of the Third International Conference on Internet Multimedia Computing and Service, Chengdu, 2011 (ACM, New York, 2011), pp. 13-16
51. S Katti, H Rahul, W Hu, D Katabi, M Médard, J Crowcroft, XORs in the air: practical wireless network coding. SIGCOMM Comput. Commun. Rev. 36(4), 243-254 (2006)

52. S Liu, Y Lin, Introduction to Grey Systems Theory, in Grey Systems, Understanding Complex Systems, vol. 68 (Springer, Berlin Heidelberg, 2011), pp. $1-18$

53. A Razzaq, A Mehaoua, Layered video transmission using wireless path diversity based on grey relational analysis, in 2011 IEEE International Conference Communications (ICC), Kyoto, June 2011 (IEEE, New York, 2011)

54. W Tu, CJ Sreenan, CT Chou, A Misra, S Jha, Resource-aware video multicasting via access gateways in wireless mesh networks, in IEEE International Conference of Network Protocols (ICNP), Orlando, October 2008 (IEEE, New York, 2008), pp. 43-52

55. T Wanqing, Efficient resource utilization for multi-flow wireless multicasting transmissions. IEEE J. Sel. Area. Comm. 30(7), 1246-1258 (2012)

56. HA Mogaibe, M Othman, NAW Abdul Hamid, S Subramaniam, On-demand channel reservation scheme for common traffic in wirelessmeshnetworks. J. Netw. Comput. Appl. 35(4), 1329-1351 (2012)

57. V Rishiwal, A Kush, S Verma, Stable and Energy Efficient Routing for Mobile Adhoc Networks, in Fifth International Conference on Information Technology: New Generations (ITNG), Las Vegas, April 2008 (IEEE, Washington, 2008), pp. 1028-1033

58. A Triviño-Cabrera, J Garcia-de-la-Nava, E Casilari, FJ Gonzàlez-Cañete, An analytical model to estimate path duration in MANETs, in Proceedings of the 9th ACM International Symposium on Modeling Analysis and Simulation of Wireless and Mobile Systems (, Torremolinos, 2006), pp. 183-186

59. W Fu, DP Agrawal, Capacity of hybrid wireless mesh networks with random APs. IEEE Trans. Mobile Comput. 12(1), 136-150 (2013)

60. I Chakeres, C Perkins, Dynamic MANET on-demand (DYMO) routing. IETF Internet Draft, Draft-ietf-manet-dymo-21. (2011)

61. J Broch, D Maltz, D Johnson, Y Jetcheva, Proceedings of the 4th annual ACM/ IEEE international conference on Mobile computing and networking, Dallas, October 1998, A performance comparison of multi-hop wireless ad hoc network routing protocols (ACM, New York, 2008), pp. 85-97

62. C Kim, Y-B Ko, NH Vaidya, Link-state routing without broadcast storming for multichannel mesh networks. Comput. Netw. 54(2), 330-340 (2010)

63. K Mase, Layer 3 wireless mesh networks: mobility management issues, Commun. Mag. IEEE 49(7), 156-163 (2011)

64. OMNET++. OMNET++, 2010. Available from: http://www.OMNETPP.org. Accessed 2012

65. H Lin, J Ma, J Hu, K Yang, PA-SHWMP: a privacy-aware secure hybrid wireless mesh protocol for IEEE $802.11 \mathrm{~s}$ wireless mesh networks. EURASIP J. Wirel. Commun. Netw. 2012, 69 (2012)

66. M Catalan-Cid, JL Ferrer, C Gomez, J Paradells, Contention- and interferenceaware flow-based routing in wireless mesh networks: design and evaluation of a novel routing metric. EURASIP J. Wirel. Commun. Netw. 2010, 1-20 (2010)

67. L lannone, K Kabassanov, S Fdida, Evaluation of cross-layer rate-aware routing in a wireless mesh network test bed. EURASIP J. Wirel. Commun. Netw. 2007(1), 42-42 (2007)

\section{doi:10.1186/1687-1499-2013-168}

Cite this article as: Barekatain et al:: GREENIE: a novel hybrid routing protocol for efficient video streaming over wireless mesh networks. EURASIP Journal on Wireless Communications and Networking 2013 2013:168.

\section{Submit your manuscript to a SpringerOpen ${ }^{\circ}$ journal and benefit from:}

- Convenient online submission

- Rigorous peer review

- Immediate publication on acceptance

- Open access: articles freely available online

- High visibility within the field

- Retaining the copyright to your article

Submit your next manuscript at $>$ springeropen.com 